\title{
NON-EXTINCTION OF A FLEMING-VIOT PARTICLE MODEL
}

\author{
MARIUSZ BIENIEK, KRZYSZTOF BURDZY AND SAM FINCH
}

\begin{abstract}
We consider a branching particle model in which particles move inside a Euclidean domain according to the following rules. The particles move as independent Brownian motions until one of them hits the boundary. This particle is killed but another randomly chosen particle branches into two particles, to keep the population size constant. We prove that the particle population does not approach the boundary simultaneously in a finite time in some Lipschitz domains. This is used to prove a limit theorem for the empirical distribution of the particle family.
\end{abstract}

\section{INTRODUCTION}

The paper is concerned with a branching particle system $\mathbf{X}_{t}=\left(X_{t}^{1}, \ldots, X_{t}^{N}\right)$ in which individual particles $X^{j}$ move as $N$ independent Brownian motions and die when they hit the complement of a fixed domain $D \subset \mathbb{R}^{d}$. To keep the population size constant, whenever any particle $X^{j}$ dies, another one is chosen uniformly from all particles inside $D$, and the chosen particle branches into two particles. Alternatively, the death/branching event can be viewed as a jump of the $j$-th particle. See Section 5 for a more detailed description of the construction.

Let $\tau_{k}$ be the time of the $k$-th jump of $\mathbf{X}_{t}$. Since the distribution of the hitting time of $\partial D$ by Brownian motion has a continuous density, only one particle can hit $\partial D$ at time $\tau_{k}$, for every $k$, a.s. The construction of the process is elementary for all $t<\tau_{\infty}=\lim _{k \rightarrow \infty} \tau_{k}$. However, there is no obvious way to continue the process $\mathbf{X}_{t}$ after the time $\tau_{\infty}$ if $\tau_{\infty}<\infty$. Hence, the question of the finiteness of $\tau_{\infty}$ is interesting. Theorem 1.1 in [10] asserts that $\tau_{\infty}=\infty$, a.s., for every domain $D$. Unfortunately, the proof of that theorem contains an irreparable error (see Example 5.3 below). The cited theorem might be true but it appears to be much harder to prove that the original incorrect argument might have suggested. Example 5.3 given below shows that result cannot be generalized to arbitrary Markov processes. Löbus ([19]) recently proved that $\tau_{\infty}=\infty$, a.s., in Euclidean domains that satisfy the internal ball condition. Another argument showing that $\tau_{\infty}=\infty$, a.s., in domains satisfying the internal ball condition is implicit in the proof of Theorem 1.4 of [10].

1991 Mathematics Subject Classification. 60J65,60J80.

Key words and phrases. Brownian motion, branching particle system.

Research supported in part by NSF Grant DMS-0600206. 
In this article, we will prove that $\tau_{\infty}=\infty$, a.s., if the domain $D \subset \mathbb{R}^{d}$ is Lipschitz with a Lipschitz constant $c$ depending on $d$ and the number $N$ of particles - see Theorem 5.1 and Remark 5.2 below. In addition, we prove theorems on existence and the form of the stationary distribution of the process $\mathbf{X}_{t}$, generalizing those in [10] - see Section 7 .

We use this attempt to rectify an error in an earlier paper to introduce two new techniques. In the end, these techniques may have greater interest or significance than the main theorems. The first technique, developed in Section 4, is the construction of a process of Brownian excursions in a cone, with all excursions starting at the vertex. Such a process exists only in cones with certain angles. The construction is combined with a coupling argument to provide a "lower bound" for $\mathbf{X}_{t}$, in an appropriate sense. The process constructed from Brownian excursions is simpler to analyze than $\mathbf{X}_{t}$.

The second technique is a new type of boundary Harnack principle (see Section 3). The standard boundary Harnack principle compares two functions satisfying a PDE with the same operator, for example, Laplacian, and different boundary conditions. Our new version of the boundary Harnack principle compares a harmonic function with a function $u$ satisfying $\Delta u=-1$. The reason for proving the new form of the boundary Harnack principle is that it allows one to compare certain probabilities and expectations, and then use a method of proof that goes back at least to Davis [12]. The "new boundary Harnack principle" has been proved independently by Atar, Athreya and Chen ([3] ), together with a number of other interesting theorems. We include a full proof of the new boundary Harnack principle because it is different from that in [3], and ours is amenable to generalizations that will be the subject of a forthcoming article.

Both techniques mentioned above - the Brownian excursion process and the boundary Harnack principle - are limited to Lipschitz domains and, moreover, the Lipschitz constant has to satisfy a certain inequality. A natural question arises whether such special Lipschitz domains are the largest natural family of sets where our results hold. It turns out that they are not. In the last section of the paper we will show that, for the two particle process, $\tau_{\infty}=\infty$, a.s., in all polyhedral domains, with arbitrary angles between the faces of the boundary. Unfortunately, our method cannot be easily adapted to the multiparticle case, so we leave this generalization as an open problem.

For some related results on Fleming-Viot type models in smooth domains, see [16] and references therein. The discrete version of the model is studied in [2]; see also references in that paper.

We are grateful to Zhenqing Chen, Davar Khoshnevisan and Yuval Peres for very helpful advice. 


\section{Preliminaries}

For $y=\left(y^{1}, \ldots, y^{d}\right) \in \mathbb{R}^{d}$, let $|y|$ denote the Euclidean norm of $y$ and let $\widetilde{y}=\left(y^{1}, \ldots, y^{d-1}\right)$. We will denote the open ball with center $x$ and radius $r$ by $B(x, r)$. The closure of a set $A$ will be denoted $\bar{A}$ and its interior will be $\operatorname{denoted} \operatorname{Int} A$. All constants, typically denoted by $c$ with or without subscript, are assumed to be strictly positive and finite.

A function $F: \mathbb{R}^{d-1} \rightarrow \mathbb{R}$ is called Lipschitz if there exists a constant $L$ such that

$$
|F(x)-F(y)| \leq L|x-y|, \quad x, y \in \mathbb{R}^{d-1} .
$$

Any constant $L$ satisfying the above condition will be called a Lipschitz constant of $F$.

Consider a bounded connected open set $D \subset \mathbb{R}^{d}, d \geq 2$. We will call $D$ a Lipschitz domain with Lipschitz constant $L$ if $\partial D$ can be covered by a finite number of open balls $B_{1}, \ldots, B_{n}$ such that for every $i=1, \ldots, n$, there exists a Lipschitz function $F_{i}: \mathbb{R}^{d-1} \rightarrow \mathbb{R}$ with Lipschitz constant $L$, and an orthonormal coordinate system $C S_{i}$ such that

$$
D \cap B_{i}=\left\{\left(y^{1}, \ldots, y^{d}\right) \text { in } C S_{i}: y^{d}>F_{i}(\widetilde{y})\right\} \cap B_{i} .
$$

The following Harnack principles can be found in [5].

Theorem 2.1 (Harnack inequality). (a) Suppose $0<r<R$. There exists $c=c(r, R, d)$ such that if $u$ is nonnegative and harmonic in $B(0, R) \subset \mathbb{R}^{d}$ and $x, y \in B(0, r)$, then

$$
u(x) \leq c u(y)
$$

(b) Suppose that $D \subset \mathbb{R}^{d}$ is a domain and $x, y \in D$ can be connected by a curve $\gamma \subset D$ such that $\inf _{z \in \gamma} \operatorname{dist}(z, \partial D) \geq R$. There exists $c=c(\gamma, R, d)$ such that if $u$ is nonnegative and harmonic in $D$, then

$$
u(x) \leq c u(y)
$$

Theorem 2.2 (Boundary Harnack principle). Suppose D is a connected Lipschitz domain. Suppose $V$ is open, $M$ is compact and $M \subset V$. Then there exists a constant $c=c(M, V, D)$ such that if $u$ and $v$ are two positive and harmonic functions on $D$ that both vanish continuously on $V \cap \partial D$, then

$$
\frac{u(x)}{v(x)} \leq c \frac{u(y)}{v(y)}, \quad x, y \in M \cap D
$$

The next theorem is a simplified version of Theorem 1 of [1].

Theorem 2.3. Assume that $D$ is a Lipschitz domain. Then there exist constants $r_{0}=$ $r_{0}(D)>0, c=c(D)<\infty$ and $a=a(D)>1$ such that if $z \in \partial D$ and $0<r \leq r_{0}$ then for all 
functions $u$ and $v$ that are bounded, positive and harmonic on $D \cap B(z$, ar $)$, and vanishing continuously on $\partial D \cap B(z$, ar $)$, we have

$$
\frac{u(x)}{v(x)} \leq c \frac{u(y)}{v(y)}, \quad x, y \in D \cap B(z, r) .
$$

Remark 2.4. Theorem 2.3 can be used to estimate the constant $c(M, V, D)$ in Theorem 2.2 as follows. Suppose that $r_{0}$ and $a$ are as in Theorem 2.3 and we can find balls $B_{i}\left(x_{i}, r_{i}\right)$, $i=1, \ldots, n$, and $B_{j}^{\prime}\left(y_{j}, \rho\right), j=1, \ldots, m, \rho>0, r_{i} \leq r_{0}, x_{i} \in \partial D, y_{j} \in D, M \subset \bigcup_{i} B_{i}\left(x_{i}, r_{i}\right) \cup$ $\bigcup_{j} B_{j}^{\prime}\left(y_{j}, \rho\right)$, and $\bigcup_{i} B_{i}\left(x_{i}, a r_{i}\right) \subset V$ and $\bigcup_{j} B_{j}^{\prime}\left(y_{j}, 2 \rho\right) \subset D$. A simple chaining argument based on Theorems 2.1 and 2.3 then shows that the constant $c(M, V, D)$ in Theorem 2.2 depends only on $n, m$ and $D$.

Next we recall some notation and results from [11]. Fix $d \geq 2$ and $p>0$. Let

$$
h(\theta)=h_{p, d}(\theta)=F(-p, p+d-2 ;(d-1) / 2 ;(1-\cos \theta) / 2),
$$

where

$$
F(a, b ; c ; x)=\sum_{k=0}^{\infty} \frac{(a)_{k}(b)_{k}}{(c)_{k} k !} x^{k}, \quad|x|<1,
$$

denotes the hypergeometric function and $(a)_{k}=a(a+1) \ldots(a+k-1),(a)_{0}=1$. The function $h$ has at least one zero in $(0, \pi)$; let $\theta_{p, d}$ denote the smallest one. The quantity $\theta_{p, d}$ is strictly decreasing in $p$ for any fixed $d \geq 2$, and strictly increasing to $\pi / 2$ in $d$ for any fixed $p>1$. In particular, if $p=2$, then

$$
h_{2, d}(\theta)=1-\frac{d}{d-1} \sin ^{2} \theta
$$

$\theta_{2, d}=\arccos \frac{1}{\sqrt{d}}$ and $\cot \theta_{2, d}=\frac{1}{\sqrt{d-1}}$. Therefore $\theta_{2,2}=\pi / 4$ and $p<2$ is equivalent to $\cot \theta_{p, d}<\frac{1}{\sqrt{d-1}}$.

For $d \geq 2$ and $p>0$ we let $\theta$ be the angle between $y$ and $(0, \ldots, 0,1)$,

$$
K_{p, d}=\left\{y \in \mathbb{R}^{d}: y \neq 0,0 \leq \theta<\theta_{p, d}\right\}
$$

and let $O$ denote the axis of $K_{p, d}$. Obviously $p<p^{\prime}$ implies $K_{p^{\prime}, d} \subset K_{p, d}$. We will drop the subscripts $p$ and $d$ and write $K$ instead of $K_{p, d}$ whenever there is no danger of confusion.

The function $v(x)=|x|^{p} h(\theta)$, where $h$ is given by (2.1), is positive and harmonic inside $K$ and continuous on $\bar{K}$ with $v(x)=0$ for $x \in \partial K$.

Let $\left(\mathbb{P}^{x}, X_{t}\right)$ be $d$-dimensional Brownian motion and for a Borel set $A \subset \mathbb{R}^{d}$ define

$$
T_{A}=\inf \left\{t>0: X_{t} \in A\right\} .
$$


Lemma 2.5. Let $F$ denote the intersection of $K=K_{p, d}$ and a hyperplane orthogonal to $O$. Let $z_{0}$ be the point of intersection of $O$ with $F$ and assume that $z_{0} \in K$. There exists $c=c(p, d)$ such that for all $z_{1}, z_{2} \in O$ with $\left|z_{0}\right|<\left|z_{1}\right|<\left|z_{2}\right|$, we have

$$
\frac{\mathbb{P}^{z_{2}}\left(T_{F}<T_{\partial K}\right)}{\mathbb{P}^{z_{1}}\left(T_{F}<T_{\partial K}\right)} \geq c\left(\frac{\left|z_{2}\right|}{\left|z_{1}\right|}\right)^{2-d-p} .
$$

Proof. Let $K_{*}$ be the unbounded component of $K \backslash F$ and

$$
u(z)=\mathbb{P}^{z}\left(T_{F}<T_{\partial K}\right), \quad z \in K_{*} .
$$

Then $u$ is positive and harmonic in $K_{*}$ and continuous on $\bar{K}_{*} \backslash(F \cap \partial K)$, with $u(z)=0$ for $z \in \partial K \backslash F$. It is easy to see that $u(x) \rightarrow 0$ as $|x| \rightarrow \infty$.

If $I(x)=x /|x|^{2}$, then the function $\widetilde{u}(x)=|x|^{2-d} u(I(x))$ is positive and harmonic in $\widetilde{K}=I\left(K_{*}\right)$ (see Lemma 1.18 of [5]). The function $\widetilde{u}$ vanishes continuously on $\partial \widetilde{K} \backslash I(F)$. Let $K^{\prime}=(1 / 2) \widetilde{K}$. Recall that $v(x)=|x|^{p} h(\theta)$ is positive and harmonic inside $K$ and continuous on $\bar{K}$ with $v(x)=0$ for $x \in \partial K$. By the boundary Harnack principle,

$$
\frac{\widetilde{u}(z)}{\widetilde{u}\left(z^{\prime}\right)} \geq c \frac{v(z)}{v\left(z^{\prime}\right)},
$$

for $z, z^{\prime} \in K^{\prime}$, where $c$ depends on $\widetilde{K}$ and $K^{\prime}$ and does not depend on $z$ and $z^{\prime}$. Note that $u(x)=|x|^{2-d} \widetilde{u}(I(x))$. Hence, for $z_{1}, z_{2} \in O \cap I\left(K^{\prime}\right)$,

$$
\frac{u\left(z_{2}\right)}{u\left(z_{1}\right)}=\frac{\left|z_{2}\right|^{2-d} \widetilde{u}\left(I\left(z_{2}\right)\right)}{\left|z_{1}\right|^{2-d} \widetilde{u}\left(I\left(z_{1}\right)\right)} \geq c \frac{\left|z_{2}\right|^{2-d} v\left(I\left(z_{2}\right)\right)}{\left|z_{1}\right|^{2-d} v\left(I\left(z_{1}\right)\right)}=c \frac{\left|z_{2}\right|^{2-d}\left|z_{2}\right|^{-p} h(0)}{\left|z_{1}\right|^{2-d}\left|z_{1}\right|^{-p} h(0)}=c\left(\frac{\left|z_{2}\right|}{\left|z_{1}\right|}\right)^{2-p-d} .
$$

The inequality holds for all $z_{1}, z_{2} \in O \cap I\left(K_{*}\right)$ (possibly with a different value of $c$ ) because the function $u$ is bounded below and above on $O \backslash I\left(K^{\prime}\right)$ by strictly positive and finite constants. This completes the proof of (2.3).

We will use the following estimate in the proof of Lemma 4.1.

Lemma 2.6. There exists a cone $K^{\prime} \subset K=K_{p, d}$ and a constant $c=c\left(K, K^{\prime}\right)$ such that for $x \in K^{\prime}$ and $t \geq|x|^{2}$,

$$
c^{-1}\left(\frac{t}{|x|^{2}}\right)^{-\frac{p}{2}} \leq \mathbb{P}^{x}\left(T_{\partial K}>t\right) \leq c\left(\frac{t}{|x|^{2}}\right)^{-\frac{p}{2}} .
$$

Proof. See [4, 6, 14] or [22]. 


\section{A BOUNDARY HARNACK PRINCIPLE}

Let $D \subset \mathbb{R}^{d}, d \geq 2$, be a bounded Lipschitz domain and let $A \subset D$ be a compact set with $\operatorname{Int} A \neq \emptyset$. For $x \in D$, define

$$
\begin{aligned}
& f(x)=\mathbb{P}^{x}\left(T_{A}<T_{\partial D}\right), \\
& g(x)=\mathbb{E}^{x} T_{\partial D} .
\end{aligned}
$$

Theorem 3.1. Assume that the Lipschitz constant $L$ of $D$ satisfies $L<\frac{1}{\sqrt{d-1}}$. Then there exists a constant $c=c(A, D)$ such that for all $x \in D$,

$$
\frac{1}{c} \leq \frac{f(x)}{g(x)} \leq c
$$

Remark 3.2. The condition $L<\frac{1}{\sqrt{d-1}}$ is sharp. See Example 3.3 below.

Proof of RHS of (3.1). Since $A$ is compact, $\inf _{x \in A} \operatorname{dist}\left(x, D^{c}\right)=c_{1}>0$. Therefore,

$$
\inf _{x \in A} \mathbb{E}^{x} T_{\partial D} \geq \inf _{x \in A} \mathbb{E}^{x} T_{\partial B\left(x, c_{1}\right)}=c_{2}>0 .
$$

By the strong Markov property applied at $T_{A}$, we have for $x \in D$,

$$
\mathbb{E}^{x} T_{\partial D} \geq c_{2} \mathbb{P}^{x}\left(T_{A}<T_{\partial D}\right)
$$

which implies the RHS of (3.1).

Proof of LHS of (3.1). Since $D$ is a bounded Lipschitz domain with Lipschitz constant $L<$ $\frac{1}{\sqrt{d-1}}$, it is easy to see that there exist $p \in(0,2)$ and $\rho>0$ with the following properties.

(i) $\operatorname{dist}(A, \partial D)>2 \rho$.

(ii) Consider any $x \in D$ with $\operatorname{dist}(x, \partial D)<\rho 2^{-5}$. Then there exists $x_{0} \in \partial D$ and an orthonormal coordinate system $C S=C S_{x_{0}}$ with the following properties. The origin of $C S$ is $x_{0}, K_{p, d} \cap B\left(x_{0}, 2 \rho\right) \subset D \cap B\left(x_{0}, 2 \rho\right)$, and $x \in O$ (that is, $x$ belongs to the axis of $K_{p, d}$ ). For $r>0$ and integer $k$, let

$$
\begin{aligned}
& E_{r}^{*}=\left\{y \in \mathbb{R}^{d} \text { in } C S:\left|\widetilde{y}-\widetilde{x}_{0}\right| \leq r \tan \left(\theta_{p, d}\right),\left|y^{d}-x_{0}^{d}\right| \leq r\right\}, \\
& \widetilde{E}_{k}=E_{2^{-k}}^{*} .
\end{aligned}
$$

We can choose $x_{0}$ and $C S$ so that for some Lipschitz function $F=F_{x_{0}}: \mathbb{R}^{d-1} \rightarrow \mathbb{R}$ with Lipschitz constant $L$, and all $k$ such that $2^{-k} \leq \rho$,

$$
D \cap \widetilde{E}_{k}=\left\{\left(y^{1}, \ldots, y^{d}\right) \text { in } C S: y^{d}>F(\widetilde{y})\right\} \cap \widetilde{E}_{k} .
$$

We fix $x \in D$ with $\operatorname{dist}(x, \partial D)<\rho 2^{-5}$ and the corresponding coordinate system $C S$ for the rest of the proof. 
Let $E_{k}=\widetilde{E}_{k} \backslash \widetilde{E}_{k+1}$ and $C_{k}=\operatorname{Int}\left(D \cap E_{k}\right)$ for $k=N_{0}, \ldots, N_{1}$, where

$$
N_{0}=\min \left\{k: 2^{-k} \leq \rho\right\}, \quad N_{1}=\max \left\{k:|x|=x^{d} \leq 2^{-k-3}\right\} .
$$

Also let $C_{N_{0}-1}=\operatorname{Int}\left(D \backslash \widetilde{E}_{N_{0}}\right)$ and $C_{N_{1}+1}=\operatorname{Int}\left(D \cap \widetilde{E}_{N_{1}+1}\right)$.

Note that $C_{i} \cap C_{j}=\emptyset$ if $i \neq j$, and $D=\bar{C}_{N_{0}-1} \cup \ldots \cup \bar{C}_{N_{1}+1}$.

Let $G(x, y)$ denote the Green function for Brownian motion killed on exiting $D$. Then

$$
g(x)=\mathbb{E}^{x} T_{\partial D}=\int_{D} G(x, y) d y=\sum_{k=N_{0}-1}^{N_{1}+1} \int_{C_{k}} G(x, y) d y .
$$

For $k=N_{0}, \ldots, N_{1}$ denote by $y_{k}$ the midpoint of the line segment being the intersection of $C_{k}$ with $x^{d}$-axis in $C S$. In other words, $\left\{y_{k}\right\}=\partial E_{(3 / 4) 2^{-k}}^{*} \cap O$. Fix $k$ and $j$ such that $j \geq 1, k \geq N_{0}, j+k \leq N_{1}$ and consider the points $y_{k}$ and $y_{k+j}$.

Let

$$
F_{k}=\bar{C}_{k} \cap \bar{C}_{k+1} \cap K_{p, d}
$$

and

$$
u(z)=\mathbb{P}^{z}\left(T_{F_{k+j}}<T_{\partial K_{p, d}}\right) .
$$

By Lemma 2.5,

$$
u\left(y_{k}\right) \geq c_{1} u\left(y_{k+j}\right)\left(\frac{2^{-k}}{2^{-k-j}}\right)^{2-p-d}=c_{1} u\left(y_{k+j}\right) 2^{j(2-p-d)},
$$

where $c_{1}=c_{1}(p, d)$. By scaling properties of Brownian motion, $u\left(y_{k+j}\right)=c_{2}=c_{2}(p, d)$, that is, $u\left(y_{k+j}\right)$ depends only on $p$ and $d$. We obtain

$$
\mathbb{P}^{z}\left(T_{F_{k+j}}<T_{\partial K_{p, d}}\right) \geq c_{3} 2^{-j(p+d-2)},
$$

where $c_{3}=c_{3}(p, d)$.

Let

$$
v(z)=\mathbb{P}^{z}\left(T_{F_{k+j}}<T_{\partial D}\right) .
$$

Note that $v\left(y_{k+j}\right) \leq 1$ and $v\left(y_{k}\right) \geq u\left(y_{k}\right) \geq c_{3} 2^{-j(p+d-2)}$, by (3.3).

We will apply Theorem 2.2 with $M=\partial E_{(3 / 4) 2^{-k-j}}^{*}$ and $V=E_{k+j}$. It follows from Remark 2.4 that the constant $c_{5}=c(M, V, D)$ may be chosen independent of $k$ and $j$. The boundary Harnack principle implies that

$$
\frac{G(x, z)}{G\left(x, y_{k+j}\right)} \geq c_{5} \frac{v(z)}{v\left(y_{k+j}\right)}
$$

for $z \in D \cap M$. The harmonic functions $G(x, \cdot)$ and $v$ have zero boundary values on $\partial D \backslash \bar{E}_{(3 / 4) 2^{-k-j}}^{*}$, so the inequality (3.4) extends to all $z \in D \backslash E_{(3 / 4) 2^{-k-j}}^{*}$, in particular, it 
applies to $z=y_{k}$. Hence,

$$
\frac{G\left(x, y_{k}\right)}{G\left(x, y_{k+j}\right)} \geq c_{5} \frac{v\left(y_{k}\right)}{v\left(y_{k+j}\right)} \geq c_{5} c_{3} 2^{-j(p+d-2)}=c_{6} 2^{-j(p+d-2)} .
$$

Now consider the function

$$
h_{m}(z)=\mathbb{P}^{z}\left(T_{\widetilde{E}_{m+2}}<T_{\partial D}\right) .
$$

By the scaling properties of Brownian motion, $h_{m}\left(y_{m}\right) \geq c_{7}>0$ for all $m=N_{0}, \ldots, N_{1}$. By the boundary Harnack principle (Theorem 2.2) applied to $u(z)=G(x, z), v(z)=h_{m}(z)$, $M=\bar{C}_{m}$ and $V=\operatorname{Int}\left(\widetilde{E}_{m-1} \backslash E_{(3 / 4) 2^{-m-1}}^{*}\right)$, we have

$$
\frac{G(x, y)}{h_{m}(y)} \leq c_{8} \frac{G\left(x, y_{m}\right)}{h_{m}\left(y_{m}\right)}
$$

for $y \in C_{m}$, where $c_{8}$ depends only on $D$, by Remark 2.4. Therefore, for $y \in C_{m}$,

$$
G(x, y) \leq c_{8} G\left(x, y_{m}\right) \frac{h_{m}(y)}{h_{m}\left(y_{m}\right)} \leq c_{8} \frac{1}{c_{7}} G\left(x, y_{m}\right)=c_{9} G\left(x, y_{m}\right) .
$$

This implies

$$
\int_{C_{k+j}} G(x, y) d y \leq c_{9} G\left(x, y_{k+j}\right) \operatorname{vol}\left(C_{k+j}\right) \leq c_{10} 2^{-d(k+j)} G\left(x, y_{k+j}\right),
$$

where $c_{10}$ depends only on $D$.

On the other hand, by the usual Harnack inequality,

$$
G(x, y) \geq c_{11} G\left(x, y_{k}\right)
$$

for $y \in B_{k}=B\left(y_{k}, 2^{-k-1}\right)$, because $B\left(y_{k}, 2^{-k-1}\right) \subset D \backslash\{x\}$. This implies that

$$
\int_{C_{k}} G(x, y) d y \geq c_{11} G\left(x, y_{k}\right) \operatorname{vol}\left(B_{k}\right)=c_{12} 2^{-k d} G\left(x, y_{k}\right)
$$

where $c_{12}$ does not depend on $k$.

Combining (3.5), (3.7) and (3.8) we have

$$
\int_{C_{k+j}} G(x, y) d y \leq c_{13} 2^{j(p-2)} \int_{C_{k}} G(x, y) d y
$$

where $c_{13}=c_{13}(D)$. Fix $q<1$. Since $p \in(0,2)$, we may choose $j$ so large that $c_{13} 2^{j(p-2)} \leq$ $q<1$. Let $a_{k}=\int_{C_{k}} G(x, y) d y$, then

$$
a_{k+j} \leq q a_{k}, \quad k=N_{0}, \ldots, N_{1}-j .
$$

Let $N_{2}=\min \left(N_{1}, N_{0}+j-1\right)$. The last inequality implies that

$$
\sum_{k=N_{0}}^{N_{1}} a_{k}=\sum_{k=N_{0}}^{N_{2}} \sum_{m=0}^{\infty} a_{k+m j} \mathbf{1}_{\left\{k+m j \leq N_{1}\right\}} \leq \sum_{k=N_{0}}^{N_{2}} \sum_{m=0}^{\infty} a_{k} q^{m}=c_{14} \sum_{k=N_{0}}^{N_{2}} a_{k} .
$$


Recall that $G(x, \cdot)$ has zero boundary values on $\partial D$, so it is bounded by $\sup _{z \in C_{N_{0}}} G(x, z)$ on the set $D \backslash \widetilde{E}_{N_{0}}$. This and (3.6) imply that $\sup _{z \in D \backslash \widetilde{E}_{N_{0}}} G(x, z) \leq c_{15} G\left(x, y_{N_{0}}\right)$. We use (3.8) to see that

$$
\begin{aligned}
a_{N_{0}-1} & =\int_{C_{N_{0}-1}} G(x, y) d y \leq c_{15} G\left(x, y_{N_{0}}\right) \operatorname{vol}\left(C_{N_{0}-1}\right) \\
& \leq c_{15} \operatorname{vol}\left(C_{N_{0}-1}\right) c_{12}^{-1} 2^{N_{0} d} \int_{C_{N_{0}}} G(x, y) d y=c_{16} a_{N_{0}} .
\end{aligned}
$$

Recall the definition of $N_{0}$ to see that $c_{16}$ depends only on $D$.

The following calculation is presented in the case $d \geq 3$ only. The case $d=2$ requires minor modifications and is left to the reader.

Let $\widetilde{G}(x, y)$ denote the Green function for Brownian motion in $\mathbb{R}^{d}$, and let $\bar{G}(x, y)$ be the Green function for Brownian motion in $B\left(x, 2^{-N_{1}-4}\right)$. It is well known that for $d \geq 3$, $\widetilde{G}(x, y)=c_{17}|x-y|^{2-d}$, where $c_{17}$ depends on $d$, and $\bar{G}(x, y)=\widetilde{G}(x, y)-\widetilde{G}(x, z)$, for $y \in B\left(x, 2^{-N_{1}-4}\right)$ and $z \in \partial B\left(x, 2^{-N_{1}-4}\right)$. It follows that for $|y-x| \leq 2^{-N_{1}-5}$,

$$
\bar{G}(x, y) \geq c_{18} \widetilde{G}(x, y) .
$$

We have $G(x, y) \leq \widetilde{G}(x, y)$ for $y \in D$, and $\int_{B(x, r)} \widetilde{G}(x, y) d y=c_{19} r^{2}$. Therefore,

$$
a_{N_{1}+1}=\int_{C_{N_{1}+1}} G(x, y) d y \leq \int_{C_{N_{1}+1}} \widetilde{G}(x, y) d y \leq \int_{B\left(x, \operatorname{diam}\left(\widetilde{E}_{N_{1}+1}\right)\right)} \widetilde{G}(x, y) d y=c_{20} 2^{-2 N_{1}} .
$$

Since $B\left(x, 2^{-N_{1}-4}\right) \subset D$,

$$
G(x, y) \geq \bar{G}(x, y)
$$

Put $y_{N_{1}+1}=\left(\widetilde{x}, x_{d}+2^{-N_{1}-5}\right)$. Then by (3.11) and (3.13),

$$
G\left(x, y_{N_{1}+1}\right) \geq c_{18} \widetilde{G}(x, y)=c_{21}\left(2^{-N_{1}}\right)^{2-d} .
$$

Moreover, by the usual Harnack inequality,

$$
G(x, y) \geq c_{22} G\left(x, y_{N_{1}+1}\right),
$$

for $y \in B\left(y_{N_{1}}, 2^{-N_{1}-2}\right)$. Therefore,

$$
\begin{aligned}
a_{N_{1}} & =\int_{C_{N_{1}}} G(x, y) d y \geq \int_{B\left(y_{N_{1}}, 2^{-N_{1}-2}\right)} G(x, y) d y \\
& \geq c_{22} G\left(x, y_{N_{1}+1}\right) \operatorname{vol}\left(B\left(y_{N_{1}}, 2^{-N_{1}-2}\right)\right) \geq c_{23}\left(2^{-N_{1}}\right)^{2-d} \cdot 2^{-N_{1} d}=c_{24} 2^{-2 N_{1}} .
\end{aligned}
$$

Combining (3.12) and (3.14), we obtain

$$
a_{N_{1}+1} \leq c_{25} a_{N_{1}}
$$


Let $C_{*}=C_{N_{0}-1} \cup \ldots \cup C_{N_{2}}$ and note that $A \subset C_{*}$. Let $\sigma_{C_{*}}=\int_{0}^{T_{\partial D}} 1_{\left\{X_{s} \in C_{*}\right\}} d s$. Then (3.9), (3.10) and (3.15) imply that

$$
\mathbb{E}^{x} T_{\partial D} \leq c_{26} \mathbb{E}^{x} \sigma_{C_{*}}
$$

Since $D$ is bounded, $\sup _{z \in D} \mathbb{E}^{z} T_{\partial D}=c_{27}<\infty$. By the strong Markov property applied at the hitting time of $C_{*}$, for $z \in D$,

$$
\mathbb{E}^{z} \sigma_{C_{*}} \leq c_{27} \mathbb{P}^{z}\left(T_{C_{*}}<T_{\partial D}\right)
$$

This and (3.16) yield

$$
\mathbb{E}^{x} T_{\partial D} \leq c_{28} \mathbb{P}^{x}\left(T_{C_{*}}<T_{\partial D}\right)
$$

Consider functions

$$
\begin{aligned}
& \xi_{1}(z)=\mathbb{P}^{z}\left(T_{A}<T_{\partial D}\right), \\
& \xi_{2}(z)=\mathbb{P}^{z}\left(T_{C_{*}}<T_{\partial D}\right) .
\end{aligned}
$$

Both functions are positive and harmonic in $D \backslash \bar{C}_{*}$, and continuous on $\bar{D} \backslash \bar{C}_{*}$ with $u(z)=$ $v(z)=0$ for $z \in \partial D \backslash \bar{C}_{*}$. We apply the boundary Harnack principle with $V=D \backslash \bar{C}_{*}$ and $M=\widetilde{E}_{N_{2}+1}$ to see that

$$
\frac{\xi_{1}(x)}{\xi_{2}(x)} \geq c_{29} \frac{\xi_{1}\left(y_{N_{2}+1}\right)}{\xi_{2}\left(y_{N_{2}+1}\right)} .
$$

We use Remark 2.4 to see that $c_{29}$ may be chosen so that it depends only on $D$. It follows from the definitions of $N_{0}, N_{2}$ and $j$ that for some constant $c_{30}$, we have $\operatorname{dist}\left(y_{N_{2}+1}, \partial D\right)>c_{30}$. This implies that $\xi_{1}\left(y_{N_{2}+1}\right)=\mathbb{P}^{y_{N_{2}+1}}\left(T_{A}<T_{\partial D}\right) \geq c_{31}$, for some $c_{31}$ depending only on $D$. We obtain from (3.18) that $\xi_{1}(x) / \xi_{2}(x) \geq c_{29} c_{31}$, and this combined with (3.17) gives

$$
\mathbb{E}^{x} T_{\partial D} \leq\left(c_{28} / c_{29} c_{31}\right) \mathbb{P}^{z}\left(T_{A}<T_{\partial D}\right) .
$$

We have proved the LHS of (3.1) for $x$ satisfying $\operatorname{dist}(x, \partial D) \leq \rho 2^{-5}$.

It is easy to check that $\inf \left\{f(x): \operatorname{dist}(x, \partial D) \geq \rho 2^{-5}\right\}>0$ and $\sup \{g(x): x \in D\}<\infty$, so the LHS of (3.1) holds for all $x \in D$.

Example 3.3. The condition $L<\frac{1}{\sqrt{d-1}}$ in Theorem 3.1 is sharp. To see this, note that for any $L>\frac{1}{\sqrt{d-1}}$ there is a $p>2$, such that the cone $K=K_{p, d}$ is a Lipschitz domain with the Lipschitz constant $L$. Let $r>0$ be such that for every $x \in O, B(x, r|x|) \subset K$. Then $g(x)=\mathbb{E}^{x} T_{\partial K} \geq \mathbb{E}^{x} T_{\partial B(x, r|x|)} \geq c_{1} r^{2}|x|^{2}$. Recall that $f(x)=\mathbb{P}^{x}\left(T_{A}<T_{\partial K}\right)$ and let $u(x)=|x|^{p} h_{p, d}(\theta)$. By the boundary Harnack principle applied to $f$ and $u$ in a neighborhood of $0, f(x) \leq c_{2}|x|^{p}$ for $x \in O,|x|<1$. Since $p>2$, we cannot have $f(x) \geq c_{3} g(x)$ in a neighborhood of 0 . The domain $K$ is unbounded but it is easy to extend the argument to $K \cap B(0,1)$. 


\section{Construction of an auxiliary process from Brownian eXCURSions}

Let $\Omega$ denote the family of all functions $\omega:[0, \infty) \rightarrow \mathbb{R}^{d} \cup\{\delta\}$ continuous up to their lifetime $R(\omega)=\inf \{t \geq 0: \omega(t)=\delta\}$ and constantly equal to $\delta$ for $t \geq R$, where $\delta$ denotes the coffin state outside $\mathbb{R}^{d}$. Let $X$ be the canonical process on $\Omega$, i.e., $X_{t}(\omega)=\omega(t)$ and let $\mathbb{P}^{x}$ denote the distribution of Brownian motion starting from $x \in \mathbb{R}^{d}$. As in (2.2), for a Borel set $A \subset \mathbb{R}^{d}$ let $T_{A}=\inf \left\{t>0: X_{t} \in A\right\}$. Let $K=K_{p, d}$ for some $p>0$, and let $X^{\prime}$ denote the process

$$
X_{t}^{\prime}= \begin{cases}X_{t}, & \text { for } t<T_{\partial K}, \\ \delta, & \text { otherwise }\end{cases}
$$

i.e., $X^{\prime}$ is the process $X$ killed on exiting $K$. If $X$ has the distribution $\mathbb{P}^{x}$, then $X^{\prime}$ is called Brownian motion in $K$ and its distribution is denoted by $\mathbb{P}_{K}^{x}$.

Let $U$ denote the family of all functions $\omega:[0, \infty) \rightarrow K \cup\{\delta\}$ such that $\omega(0)=0$, continuous up to their lifetime $R$. Let $H^{0}$ denote a standard excursion law of Brownian motion in $K_{p, d}$ starting from 0 . Namely, $H^{0}$ is a nonnegative and $\sigma$-finite measure on $\Omega$ such that $X$ is strong Markov under $H^{0}$ with the $\mathbb{P}_{K}$ transition probabilities and $H^{0}\left(\lim _{t \rightarrow 0} X_{t} \neq 0\right)=0$. We have $H^{0}(\Omega \backslash U)=0$. The existence of $H^{0}$ follows from results of [20] and [8].

Lemma 4.1. There exists $c \in(0, \infty)$ such that

$$
H^{0}(R>t)=c t^{-\frac{p}{2}}, \quad t>0 .
$$

Proof. Let $y_{\varepsilon}=(0, \ldots, 0, \varepsilon) \in \mathbb{R}^{d}$ and let $G_{K}(x, y)$ denote the Green function for $K$. By Theorem 4.1 of [8],

$$
H^{0}(R>t)=c_{1} \lim _{\substack{z \rightarrow 0 \\ z \in K}} \frac{\mathbb{P}^{z}\left(T_{\partial K}>t\right)}{G_{K}\left(z, y_{1}\right)} .
$$

By Theorem 2.2 of [8], which is an improvement of the boundary Harnack principle, there exists $c(K, \varepsilon)$ such that for all functions $h_{1}$ and $h_{2}$ which are positive and harmonic in $K$ and vanish continuously on $\partial K$, we have

$$
c(K, \varepsilon)^{-1} \frac{h_{1}(y)}{h_{2}(y)} \leq \frac{h_{1}(x)}{h_{2}(x)} \leq c(K, \varepsilon) \frac{h_{1}(y)}{h_{2}(y)},
$$

for all $x, y \in K \cap B(0, \varepsilon)$, and $\lim _{\varepsilon \rightarrow 0} c(K, \varepsilon)=1$. Therefore, the limit

$$
\lim _{\substack{z \rightarrow 0 \\ z \in K}} \frac{h_{1}(z)}{h_{2}(z)}
$$

exists and belongs to $(0, \infty)$ for all functions $h_{1}, h_{2}$ satisfying the above assumptions. We apply this claim to $h_{1}(z)=G_{K}\left(z, y_{1}\right)$ and $h_{2}(z)=|z|^{p} h(\theta)$, to conclude that

$$
\lim _{\varepsilon \rightarrow 0} \frac{G_{K}\left(y_{\varepsilon}, y_{1}\right)}{\varepsilon^{p}}=c \in(0, \infty)
$$


and

$$
H^{0}(R>t)=c \lim _{\varepsilon \rightarrow 0} \frac{\mathbb{P}^{y_{\varepsilon}}\left(T_{\partial K}>t\right)}{\varepsilon^{p}}
$$

By Lemma 2.6,

$$
c^{-1} t^{-\frac{p}{2}} \leq \frac{\mathbb{P}^{y_{\varepsilon}}\left(T_{\partial K}>t\right)}{\varepsilon^{p}} \leq c t^{-\frac{p}{2}}
$$

for $t \geq \varepsilon^{2}$ which implies $c^{-1} t^{-\frac{p}{2}} \leq H^{0}(R>t) \leq c t^{-\frac{p}{2}}$, for $t \geq 0$. Therefore $H^{0}(R>1)$ is a positive and finite number.

Now mimicking the proof of Proposition 5.1 of [8], using (4.3) instead of (4.2), we easily see that if $\{X(t), t \geq 0\}$ has the distribution $H^{0}$, then for every $a>0$ the scaled process $\{\sqrt{a} X(t / a), t \geq 0\}$ has the distribution $a^{p / 2} H^{0}$. In particular, for every $a>0$

$$
H^{0}(R>t)=a^{p / 2} H^{0}(R>a t), \quad t \geq 0,
$$

and putting $a=1 / t$ we obtain (4.1) with $c=H^{0}(R>1)$.

Let $\lambda$ denote the Lebesgue measure on $\mathbb{R}_{+}=[0, \infty)$ and let $\mathcal{P}$ be a Poisson point process on $\mathbb{R}_{+} \times U$ with characteristic measure $\lambda \times H^{0}$, i.e., $\mathcal{P}$ is a random subset of $\mathbb{R}_{+} \times U$ such that for every pair $A_{1}, A_{2}$ of disjoint nonrandom subsets of $\mathbb{R}_{+} \times U, \operatorname{card}\left(\mathcal{P} \cap A_{1}\right)$ and $\operatorname{card}\left(\mathcal{P} \cap A_{2}\right)$ are independent random variables with Poisson distributions with means $\left(\lambda \times H^{0}\right)\left(A_{1}\right)$ and $\left(\lambda \times H^{0}\right)\left(A_{2}\right)$, respectively $([18])$. With probability 1 , there are no two points with the same first coordinate, and therefore the elements of $\mathcal{P}$ may be unambiguously denoted by $\left(t, e_{t}\right)$. Let

$$
R_{t}=\inf \left\{s>0: e_{t}(s)=\delta\right\}
$$

By abuse of notation, for a generic element $e$ of $U$ we will write

$$
R(e)=\inf \{s>0: e(s)=\delta\} .
$$

Lemma 4.2. If $p \in(0,2)$, then for every $s>0$,

$$
\sum_{t \leq s} R_{t}<\infty, \quad \text { a.s. }
$$

Proof. We use Theorem 4.6 of [18]: if $\varphi: \mathbb{R}_{+} \times U \rightarrow \mathbb{R}_{+}$is a measurable function, then

$$
\sum_{t} \varphi\left(t, e_{t}\right)<\infty, \quad \text { a.s. }
$$

iff

$$
\iint_{\mathbb{R}_{+} \times U}(\varphi(t, e) \wedge 1) d t H^{0}(d e)<\infty .
$$

In particular, if $\varphi(t, e)=R(e) 1_{[0, s]}(t)$, then

$$
\sum_{t \leq s} R_{t}<\infty, \quad \text { a.s. }
$$


iff

$$
\iint_{[0, s] \times U}(R(e) \wedge 1) d t H^{0}(d e)<\infty .
$$

If we let $U^{-}=\{e \in U: R(e) \leq 1\}$ and $U^{+}=\{e \in U: R(e)>1\}$ then

$$
\iint_{[0, s] \times U}(R(e) \wedge 1) d t H^{0}(d e)=s \int_{U^{-}} R(e) H^{0}(d e)+s H^{0}\left(U^{+}\right) .
$$

By Lemma 4.1,

$$
H^{0}\left(U^{+}\right)=\int_{1}^{\infty} H^{0}(R \in d t)=c \int_{1}^{\infty} t^{-p / 2-1} d t<\infty,
$$

because $p>0$, and

$$
\int_{U^{-}} R d H^{0}=\int_{0}^{1} t H^{0}(R \in d t)=c \int_{0}^{1} t \cdot t^{-p / 2-1} d t<\infty,
$$

because $p<2$.

By Lemma 4.2, the following process $Z$ with values in $K_{p, d} \cup\{0\}$, where $p \in(0,2)$, is well defined. For every $t>0$ the formula

$$
t= \begin{cases}s+\sum_{u<r} R_{u}, & \text { if there exists } r<t \text { such that } \sum_{u<r} R_{u}<t \leq \sum_{u \leq r} R_{u}, \\ \sum_{u<r} R_{u}, & \text { otherwise }\end{cases}
$$

defines a unique pair $(r, s)$ with $r>0$ and $s \in\left[0, R_{r}\right)$ (in the first case). Then we define

$$
Z_{t}= \begin{cases}e_{r}(t), & \text { if there exists } r<t \text { such that } \sum_{u<r} R_{u}<t<\sum_{u \leq r} R_{u} \\ 0, & \text { otherwise. }\end{cases}
$$

The process $Z$ takes values in $K \cup\{0\}$. Let $\sigma_{t}=\sum_{s \leq t} R_{t}$ for $t \geq 0$.

Lemma 4.3. The process $\sigma$ is a stable subordinator with index $p / 2$.

Proof. The process $\sigma$ is increasing and has values in $[0, \infty)$. Its paths are right-continuous with left limits. Note that $\left\{\left(t, R\left(e_{t}\right)\right)\right\}_{e \in \mathcal{P}}$ is a Poisson point process on $\mathbb{R}_{+} \times \mathbb{R}_{+}$with characteristic measure $\lambda \times \Pi$, where $\Pi$ is given by

$$
\Pi(d x)=H^{0}(R \in d x)=c x^{-p / 2-1} d x,
$$

where the last formula follows from Lemma 4.1. This implies that $\sigma$ is a process with independent and stationary increments, so $\sigma$ is a Lévy process. Moreover $\sigma$ is a subordinator, 
since it has values in $[0, \infty)$ only. We use calculations that can be found in Section 0.5 and on page 73 of [7] to see that the Laplace transform of $\sigma$ is

$$
\begin{aligned}
E \exp \left(-\lambda \sigma_{t}\right) & =\exp \left\{-t \int_{0}^{\infty}\left(1-\mathrm{e}^{-\lambda x}\right) \Pi(d x)\right\} \\
& =\exp \left\{-c t \int_{0}^{\infty}\left(1-\mathrm{e}^{-\lambda x}\right) x^{-p / 2-1} d x\right\} \\
& =\exp \left(-c t \lambda^{p / 2}\right) .
\end{aligned}
$$

Therefore $\sigma$ is stable with index $p / 2$.

\section{Construction of a Fleming-Viot process}

We recall the following informal description of a Fleming-Viot-type particle system from [10]. Consider an open set $D \subset \mathbb{R}^{d}$ and an integer $N \geq 2$. Let $\mathbf{X}_{t}=\left(X_{t}^{1}, \ldots, X_{t}^{N}\right)$ be a process with values in $D^{N}$ defined as follows. Let $\mathbf{X}_{0}=\left(x^{1}, \ldots, x^{N}\right) \in D^{N}$. Then the processes $X_{t}^{1}, \ldots, X_{t}^{N}$ evolve as independent Brownian motions until the time $\tau_{1}$ when one of them, say, $X^{j}$ hits the boundary of $D$. At this time one of the remaining particles is chosen uniformly, say, $X^{k}$, and the process $X^{j}$ jumps at time $\tau_{1}$ to $X_{\tau_{1}}^{k}$. The processes $X_{t}^{1}, \ldots, X_{t}^{N}$ continue evolving as independent Brownian motions after time $\tau_{1}$ until the first time $\tau_{2}>\tau_{1}$ when one of them hits the boundary of $D$. Again at the time $\tau_{2}$ the particle which approaches the boundary jumps to the current location of a particle chosen uniformly at random from amongst the ones strictly inside $D$. The subsequent evolution of $\mathbf{X}$ proceeds in the same way. The above recipe defines the process $\mathbf{X}_{t}$ only for $t \leq \tau_{\infty}=\lim _{k \rightarrow \infty} \tau_{k}$. There is no natural way to define the process $\mathbf{X}_{t}$ for $t>\tau_{\infty}$. Hence, it is a natural problem to determine whether $\tau_{\infty}=\infty$, a.s.

Theorem 5.1. There exists a constant $c=c(N, d)$ such that if $D \subset \mathbb{R}^{d}$ is a bounded Lipschitz domain with the Lipschitz constant $L<c(N, d)$, then $\tau_{\infty}=\infty$, a.s. Moreover, $c(N, d)$ increases in $N$, decreases in $d$ and

$$
\lim _{N \rightarrow \infty} c(N, d)=c(d)=\frac{1}{\sqrt{d-1}} .
$$

Proof. First note that $\tau_{\infty}$ is finite if and only if all the processes $X_{t}^{1}, \ldots, X_{t}^{N}$ hit $\partial D$ at the same time, so we need to prove that this is impossible. The idea of the proof is to construct processes $Y_{t}^{1}, \ldots, Y_{t}^{N}$ which are easy to analyze, with values in $\bar{D}$ and such that for every $1 \leq j \leq N$

$$
\left\{t: X_{t}^{j} \in \partial D\right\} \subset\left\{t: Y_{t}^{j} \in \partial D\right\} \stackrel{\text { df }}{=} A_{j}
$$

Then we will prove that that $A_{1} \cap \ldots \cap A_{N}=\emptyset$, a.s. 
Recall the definition of $\theta_{p, d}$ and $K_{p, d}$. Let $p^{\prime}=2-2 / N$ and

$$
c(N, d)=\cot \theta_{p^{\prime}, d}
$$

Define

$$
D_{r}=\{x \in D: \operatorname{dist}(x, \partial D) \geq r\} .
$$

Since $D$ is Lipschitz, there exists a small $r>0$ for which the following is true. For every $x \in \overline{D \backslash D_{r}}$ there exist an orthonormal coordinate system $C S_{x}, y_{x} \in \partial D$ and a Lipschitz function $F_{x}: \mathbb{R}^{d-1} \rightarrow \mathbb{R}$ such that $y_{x}$ is the origin of $C S_{x}$ and

$$
D \cap B\left(y_{x}, r\right) \subset\left\{y \text { in } C S_{x}: y^{d}>F_{x}(\widetilde{y})\right\} \cap B\left(y_{x}, r\right)
$$

Moreover, since $L<c(N, d)$, we can choose $y_{x}$ and $C S_{x}$ so that we can find a cone $K_{x}$ with vertex $y_{x}$ and axis passing through $x$ which can be described in $C S_{x}$ as $K_{p, d}$ with $p<p^{\prime}$, and such that

$$
K_{p^{\prime}, d} \cap B\left(y_{x}, r\right) \subset K_{p, d} \cap B\left(y_{x}, r\right) \subset D \cap B\left(y_{x}, r\right) .
$$

Next we will present a very special construction of the process $\mathbf{X}_{t}$, based on a family of independent Brownian motions. We need this construction to show independence of processes $Y^{1}, Y^{2}, \ldots, Y^{N}$, to be constructed in a subsequent step.

We fix $j \in\{1, \ldots, N\}$ and let $\tau_{k}^{j}$ denote the time of the $k$-th jump of $X_{t}^{j}$. We represent the evolution of $X^{j}$ on the interval $\left[0, \tau_{1}^{j}\right)$ as follows. We start with a family of independent Brownian motions $\widetilde{W^{0}}, \widetilde{W^{1}}, \widetilde{W}^{2}, \ldots$ in $\mathbb{R}^{d}$ starting from 0. Suppose that $X_{0}^{j}=x_{0} \in D \backslash D_{r / 2}$. The argument needs only minor modifications if $x_{0} \in D_{r / 2}$. Let $W^{0}=\widetilde{W}^{0}+x_{0}$ and consider the cone $K_{x_{0}}$ defined as above. Let

$$
\begin{aligned}
& \sigma_{1}^{\prime}=\inf \left\{t>0: W_{t}^{0} \in \partial K_{x_{0}}\right\}, \\
& \sigma_{1}^{\prime \prime}=\sigma_{1}^{\prime} \wedge \inf \left\{t>0: W_{t}^{0} \notin D_{r}\right\}, \\
& \sigma_{1}= \begin{cases}\sigma_{1}^{\prime}, & \text { if } \sigma_{1}^{\prime \prime}=\sigma_{1}^{\prime}, \\
\inf \left\{t>\sigma_{1}^{\prime \prime}: W_{t}^{0} \notin D_{r / 2}\right\}, & \text { if } \sigma_{1}^{\prime \prime}<\sigma_{1}^{\prime} .\end{cases}
\end{aligned}
$$

Inductively, for $n \geq 1$, given $x_{n}=W_{\sigma_{n}}^{n-1}$ we define the cone $K_{x_{n}}$, let $W^{n}=\widetilde{W}^{n}+x_{n}$, and

$$
\begin{aligned}
\sigma_{n+1}^{\prime} & =\inf \left\{t>0: W_{t}^{n} \in \partial K_{x_{n}}\right\}, \\
\sigma_{n+1}^{\prime \prime} & =\sigma_{n+1}^{\prime} \wedge \inf \left\{t>0: W_{t}^{n} \notin D_{r}\right\}, \\
\sigma_{n+1} & = \begin{cases}\sigma_{n+1}^{\prime}, & \text { if } \sigma_{n+1}^{\prime \prime}=\sigma_{n+1}^{\prime}, \\
\inf \left\{t>\sigma_{n+1}^{\prime \prime}: W_{t}^{n} \notin D_{r}\right\}, & \text { if } \sigma_{n+1}^{\prime \prime}<\sigma_{n+1}^{\prime} .\end{cases}
\end{aligned}
$$


Then we define $T_{0}=0$,

$$
T_{n}=\tau_{1}^{j} \wedge \sum_{k=1}^{n} \sigma_{k}, \quad n \geq 1
$$

and

$$
X_{t}^{j}=W_{t}^{k}, \quad \text { for } T_{k-1}<t \leq T_{k}, \quad k=0,1,2, \ldots
$$

This procedure represents $X^{j}$ on the interval $\left[0, \tau_{1}^{j}\right)$. Strictly speaking, $\tau_{1}^{j}$ is defined in terms of $\widetilde{W}^{0}, \widetilde{W}^{1}, \widetilde{W}^{2}, \ldots$

The complete construction of $\mathbf{X}_{t}$ on the interval $\left[0, \tau_{\infty}\right)$ requires that we start with a family $\left\{\widetilde{W}^{j, k, n}\right\}_{1 \leq j \leq N, k \geq 0, n \geq 0}$ of independent Brownian motions. For fixed $j$ and $k$, the subfamily $\left\{\widetilde{W}^{j, k, n}\right\}_{n \geq 0}$ is used to construct $X^{j}$ on the interval $\left[\tau_{k}^{j}, \tau_{k+1}^{j}\right)$, according to the recipe described above. The whole procedure is straightforward and elementary but tedious to describe so we leave the details to the reader.

Let $\left\{\widetilde{Z}^{j, k, m, n}\right\}_{1 \leq j \leq N, k \geq 0, m \geq 0, n \geq 0}$ be a family of independent copies of the process $Z$ defined in (4.4), independent of $\left\{\widetilde{W}^{j, k, n}\right\}_{1 \leq j \leq N, k \geq 0, n \geq 0}$. We will present a construction of $Y^{j}$ on the interval $\left[T_{0}, T_{1}\right) \subset\left[0, \tau_{1}^{j}\right)$, based on $X^{j}$ and $\left\{\widetilde{Z}^{j, 0,0, n}\right\}_{n \geq 0}$. For any fixed $j, k$ and $m$, we can construct $Y^{j}$ on $\left[T_{m}, T_{m+1}\right) \subset\left[\tau_{k}^{j}, \tau_{k+1}^{j}\right)$ using $X^{j}$ and $\left\{\widetilde{Z}^{j, k, m, n}\right\}_{n \geq 0}$ in an analogous way. (Strictly speaking, we should not speak about $\left[T_{m}, T_{m+1}\right) \subset\left[\tau_{k}^{j}, \tau_{k+1}^{j}\right.$ ) but about a subinterval of $\left[\tau_{k}^{j}, \tau_{k+1}^{j}\right)$ constructed in a way analogous to $\left[T_{m}, T_{m+1}\right)$.)

Let $\mathcal{T}_{n}$ be the isometry that maps $K_{p, d}$ onto $K_{x_{n}}$ and let $Z^{n}=\mathcal{T}\left(\widetilde{Z}^{j, 0,0, n}\right)$. We introduce a moving cone $C_{t}^{n}$ with the vertex $X_{t}^{j}$ and the axis parallel to the axis of $K_{x_{n}}$, but directed downwards, i.e., we put $C_{*}^{n}(x)=x-K_{x_{n}}$ and $C_{t}^{n}=C_{*}^{n}\left(X_{t}^{j}\right), T_{n} \leq t<T_{n+1}$. Let

$$
\begin{gathered}
S_{1}=\sigma_{1}^{\prime \prime} \wedge \inf \left\{t>0: Z_{t}^{1} \in \partial C_{t}^{0}\right\}, \\
Y_{t}^{(1)}=X_{t}-X\left(S_{1}\right)+Z^{1}\left(S_{1}\right), \quad t \geq S_{1}, \\
R_{1}=\sigma_{1}^{\prime \prime} \wedge \inf \left\{t>S_{1}: Y_{t}^{(1)} \in \partial K_{x_{0}}\right\} .
\end{gathered}
$$

By definition, $S_{1} \leq T_{1}$. Then we define

$$
Y_{t}^{j}= \begin{cases}Z_{t}^{1}, & \text { for } t \in\left[0, S_{1}\right), \\ Y_{t}^{(1)}, & \text { for } t \in\left[S_{1}, R_{1}\right) .\end{cases}
$$

For $n \geq 2$, we define

$$
\begin{gathered}
S_{n}=\sigma_{1}^{\prime \prime} \wedge \inf \left\{t>R_{n-1}: Z^{n}\left(t-R_{n-1}\right) \in \partial C_{t}^{n-1}\right\}, \\
Y_{t}^{(n)}=X_{t}-X\left(S_{n}\right)+Z^{n}\left(S_{n}-R_{n-1}\right), \quad t \geq S_{n}, \\
R_{n}=\sigma_{1}^{\prime \prime} \wedge \inf \left\{t>S_{n}: Y_{t}^{(n)} \in \partial \bar{K}_{x_{0}}\right\},
\end{gathered}
$$


and

$$
Y_{t}^{j}= \begin{cases}Z^{n}\left(t-R_{n-1}\right), & \text { for } t \in\left[R_{n-1}, S_{n}\right), \\ Y_{t}^{(n)}, & \text { for } t \in\left[S_{n}, R_{n}\right) .\end{cases}
$$

We continue this process until the time $\sigma_{1}^{\prime \prime}$ and then we put $Y_{t}^{j}=Y_{\sigma_{1}^{\prime \prime}}^{j}$ for $t \in\left[\sigma_{1}^{\prime \prime}, \sigma_{1}\right)$.

By construction, processes $X^{1}, \ldots, X^{N}$ and $Y^{1}, \ldots, Y^{N}$ satisfy (5.2). Moreover, independence of all processes in the family $\left\{\widetilde{Z}^{j, k, m, n}\right\}_{1 \leq j \leq N, k \geq 0, m \geq 0, n \geq 0} \cup\left\{\widetilde{W}^{j, k, n}\right\}_{1 \leq j \leq N, k \geq 0, n \geq 0}$ implies that processes $Y^{1}, \ldots, Y^{N}$ are independent. It remains to prove that, a.s.,

$$
A_{1} \cap \ldots \cap A_{N}=\emptyset .
$$

Recall that $A_{j}=\left\{t \geq 0: Y^{j} \in \partial D\right\}$. The construction of $Y^{j}$ from independent pieces of processes analogous to $Z$ suggests that $A_{j}$ is the range of a stable subordinator, because of Lemma 4.3. The matter is slightly complicated by the fact that $Y_{t}^{j}=Y_{\sigma_{1}^{\prime \prime}}^{j}$ for $t \in\left[\sigma_{1}^{\prime \prime}, \sigma_{1}\right)$, and similarly for other analogous intervals. To deal with this problem, we introduce the following sequence of stopping times, $U_{0}=0$,

$$
\begin{aligned}
& U_{k}^{*}=\inf \left\{t \geq U_{k}: \max _{1 \leq j \leq N} \operatorname{dist}\left(X_{t}^{j}, \partial D\right) \geq r\right\}, \quad k \geq 0, \\
& U_{k}=\inf \left\{t \geq U_{k-1}^{*}: \max _{1 \leq j \leq N} \operatorname{dist}\left(X_{t}^{j}, \partial D\right) \leq r / 2\right\}, \quad k \geq 1 .
\end{aligned}
$$

On each interval $\left(U_{k}, U_{k}^{*}\right]$, sets $A_{1}, \ldots, A_{N}$ are independent and each one has the same distribution as the range of a stable subordinator with index $p / 2$. It will suffice to prove that for each fixed $k$, a.s.,

$$
\left(U_{k}, U_{k}^{*}\right] \cap A_{1} \cap \ldots \cap A_{N}=\emptyset .
$$

We use the following result of Hawkes [17]: The ranges of two independent stable subordinators with indices $\alpha$ and $\beta$ intersect if and only if $\alpha+\beta>1$, in which case the intersection is stochastically equivalent to the range of a stable subordinator of index $\alpha+\beta-1$. Therefore, by induction, (5.4) holds if and only if $\frac{N p}{2}-N+1<0$. This condition holds since $p<p^{\prime}=2-\frac{2}{N}$.

Remark 5.2. Let $D \subset \mathbb{R}^{d}$ be a bounded Lipschitz domain with the Lipschitz constant $L<$ $\frac{1}{\sqrt{d-1}}$. Then by (5.1) we see that there exists $N_{0}$ so large that $L<c(N, d)$ for all $N \geq N_{0}$. In consequence, the Fleming-Viot-type particle process $\mathbf{X}_{t}$ in $D$ is well defined for all $t \geq 0$ provided it consists of $N$ particles with $N \geq N_{0}$.

Example 5.3. The proof of Theorem 1.1 in [10] contains an error. Formula (2.1) in [10] does not follow "by induction" from the previous statement. We will show that the error is irreparable in the following sense. The proof of Theorem 1.1 in [10] is based only on two properties of Brownian motion - the strong Markov property and the fact the the hitting 
time distribution of a compact set has no atoms (assuming that the starting point lies outside the set). Hence, if some version of that argument were true, it would apply to almost all nontrivial examples of Markov processes with continuous time, and in particular to all diffusions. However we may find a diffusion for which the analogue of Theorem 1.1 in [10] is false. Let $X_{t}$ be the diffusion on $[0, \infty)$, started at $X_{0}=1$ and satisfying the SDE

$$
d X_{t}=d W_{t}-\frac{5}{2 X_{t}} d t
$$

We make 0 absorbing so that it can play the role of the boundary for the domain $D=(0, \infty)$. Notice that although $X_{t}$ is not a Bessel process, as we have reversed the drift term, it scales in the same way. That is, for $\alpha>0, \alpha X_{t \alpha^{-2}}$ is a diffusion satisfying the same SDE, but started at $\alpha$. Let $\mathbf{Y}_{t}^{i}=\left(Y_{t}^{i, 1}, Y_{t}^{i, 2}\right), i=1 \ldots \infty$, be a double sequence of independent copies of $X_{t}$, and set

$$
\begin{aligned}
& \sigma_{i}=\inf \left\{t>0: Y_{t}^{i, 1} \wedge Y_{t}^{i, 2}=0\right\}, \\
& \alpha_{i}=Y_{\sigma_{i}}^{i, 1} \vee Y_{\sigma_{i}}^{i, 2} .
\end{aligned}
$$

Now, construct a two-particle Fleming-Viot type process $\mathbf{X}_{t}=\left(X_{t}^{1}, X_{t}^{2}\right)$ as follows. First let $\tau_{1}=\sigma_{1}$ and set $\mathbf{X}_{t}=\mathbf{Y}_{t}^{1}$ for $t \in\left[0, \tau_{1}\right)$. At $\tau_{1}$ one of the particles hits the boundary and jumps to $\xi_{1}=\alpha_{1}$. To continue the process we use the scaling property of $\mathbf{Y}_{t}$ and set $\mathbf{X}_{t}=\xi_{1} \mathbf{Y}_{\left(t-\tau_{1}\right) \xi_{1}^{-2}}^{2}$ for $t \in\left[\tau_{1}, \tau_{2}\right)$ where $\tau_{2}=\tau_{1}+\xi_{1}{ }^{2} \sigma_{2}$. At $\tau_{2}$ a second particle hits the boundary and jumps, this time to $\xi_{2}=\alpha_{2} \xi_{1}$, and we continue the process in the same way by setting

$$
\begin{aligned}
& \xi_{i}=\prod_{j=1}^{i} \alpha_{j}, \quad \tau_{i}=\sum_{j=1}^{i} \xi_{j-1}{ }^{2} \sigma_{j}, \\
& \mathbf{X}_{t}=\xi_{i} \mathbf{Y}_{\left(t-\tau_{i}\right) \xi_{i}{ }^{-2}}, \quad \text { for } t \in\left[\tau_{i}, \tau_{i+1}\right) .
\end{aligned}
$$

Then $\mathbf{X}_{t}$ evolves as two independent copies of $X_{t}$ with Fleming-Viot type jumps when a particle hits the boundary. The process $\mathbf{X}_{t}$ is well defined up until $\tau_{\infty}$ and if the analogue of [10, Theorem 1.1] were to hold for this process we would have $\tau_{\infty}=\infty$ almost surely. In fact the opposite is true. We will show now that $\mathbb{E} \tau_{\infty}<\infty$ and hence $\tau_{\infty}<\infty$ almost surely. To do this it will be sufficient to show $\mathbb{E}\left(\alpha_{1}^{2}\right)<1$ and $\mathbb{E} \sigma_{1}<\infty$. Let $f(x, y)=$ $x^{4}+y^{4}-x^{2} y^{2}$ and notice $f(x, x)=f(x, 0)=f(0, x)=x^{4}$. We may check using Ito's formula that $f\left(Y_{t \wedge \sigma_{i}}^{i, 1}, Y_{t \wedge \sigma_{i}}^{i, 2}\right)$ is a positive local martingale and hence a supermartingale. By the optional stopping theorem

$$
\mathbb{E}\left(\alpha_{1}^{4}\right)=\mathbb{E} f\left(Y_{\sigma_{1}}^{1,1}, Y_{\sigma_{1}}^{1,2}\right) \leq \mathbb{E} f\left(Y_{0}^{1,1}, Y_{0}^{1,2}\right)=1
$$


Furthermore, $\alpha_{1}$ is not almost surely constant and so by Jensen's inequality

$$
\mathbb{E}\left(\alpha_{1}^{2}\right)<\sqrt{\mathbb{E}\left(\alpha_{1}^{4}\right)}=1 .
$$

We may use Ito's formula again to show that $X_{t}^{2}+4 t$ is a local martingale and so by the optional stopping theorem again we have that $\mathbb{E}\left(\sigma_{1}\right) \leq \frac{1}{4}$.

By independence of the $\mathbf{Y}^{i}$ processes we have that $\mathbb{E}\left(\xi_{i}{ }^{2}\right)=\mathbb{E}\left(\alpha_{1}{ }^{2}\right)^{i}$ and so

$$
\mathbb{E} \tau_{\infty}=\sum_{j=1}^{\infty} \mathbb{E}\left(\xi_{j-1}{ }^{2} \sigma_{j}\right) \leq \frac{1}{4} \sum_{j=0}^{\infty} \mathbb{E}\left(\alpha_{1}^{2}\right)^{j}<\infty .
$$

\section{Hitting PRobabilities of COMPACT SETS}

This section is devoted to a technical estimate needed in the proof of Theorem 7.1. Recall definitions of $D_{r}$ and $\mathbf{X}_{t}=\left(X_{t}^{1}, \ldots, X_{t}^{N}\right)$.

Lemma 6.1. Fix $N \geq 2$ and let $D \subset \mathbb{R}^{d}$ be a bounded Lipschitz domain with the Lipschitz constant $L<c(N, d)$.

(i) For any fixed $k \in\{1, \ldots, N\}$, and for every $r>0$ such that $\operatorname{Int} D_{r} \neq \emptyset$, there exist $c>0$ and $t>0$ such that for all $\mathbf{x} \in D^{N}$,

$$
\mathbb{P}^{\mathbf{x}}\left(X_{t}^{k} \in D_{r}\right) \geq c
$$

(ii) For every $r>0$ such that $\operatorname{Int} D_{r} \neq \emptyset$, there exist $c>0$ and $t>0$ such that for all $\mathbf{x} \in D^{N}$,

$$
\mathbb{P}^{\mathbf{x}}\left(\mathbf{X}_{t} \in D_{r}^{N}\right) \geq c
$$

Proof. (i) Fix $r>0$ such that $\operatorname{Int} D_{r} \neq \emptyset$. Recall that notation such as $T_{D_{r}}, T_{\partial D}$, etc. refers to hitting times by Brownian motion. By Theorem 3.1 there exists $c_{0}=c_{0}(r)$ such that for all $x \in D$,

$$
\mathbb{P}^{x}\left(T_{D_{r}}<T_{\partial D}\right) \geq c_{0} \mathbb{E}^{x} T_{\partial D}
$$

Fix $k$ and let $T_{D_{r}}^{X^{k}}=\inf \left\{t \geq 0: X_{t}^{k} \in D_{r}\right\}$, and

$$
Y_{t}=X^{k}\left(t \wedge T_{D_{r}}^{X^{k}}\right)
$$

Define $T_{0}=0$ and

$$
T_{n+1}=\inf \left\{t>T_{n}: \lim _{s \rightarrow t^{-}} Y_{s} \in \partial D\right\} \wedge T_{D_{r}}^{X^{k}} .
$$

Let $M_{0}=0$ and

$$
M_{n}=\frac{1}{c_{0}} \mathbf{1}_{\left\{Y\left(T_{n}\right) \in D_{r}\right\}}-T_{n}, \quad n \geq 1,
$$

and

$$
\mathcal{F}_{n}=\sigma\left(\mathbf{X}_{t}, t \leq T_{n}\right)
$$


It is easy to see that $E T_{n}<\infty$ so $E\left|M_{n}\right|<\infty$. For $\mathbf{x}=\left(x_{1}, x_{2}, \ldots, x_{N}\right) \in D^{N}$ with $x_{k} \notin D_{r}$,

$$
\begin{aligned}
& \mathbb{E}^{\mathbf{x}}\left(M_{n+1}-M_{n} \mid \mathcal{F}_{n}\right) \\
& =\frac{1}{c_{0}} \mathbb{E}^{\mathbf{x}}\left(\mathbf{1}_{\left\{Y\left(T_{n+1}\right) \in D_{r}\right\}}\left(\mathbf{1}_{\left\{Y\left(T_{n}\right) \notin D_{r}\right\}}+\mathbf{1}_{\left\{Y\left(T_{n}\right) \in D_{r}\right\}}\right)-\mathbf{1}_{\left\{Y\left(T_{n}\right) \in D_{r}\right\}} \mid \mathcal{F}_{n}\right)-\mathbb{E}^{\mathbf{x}}\left(T_{n+1}-T_{n} \mid \mathcal{F}_{n}\right) \\
& =\frac{1}{c_{0}} \mathbb{E}^{\mathbf{x}}\left(\mathbf{1}_{\left\{Y\left(T_{n+1}\right) \in D_{r}\right\}} \mathbf{1}_{\left\{Y\left(T_{n}\right) \notin D_{r}\right\}}+\mathbf{1}_{\left\{Y\left(T_{n}\right) \in D_{r}\right\}}-\mathbf{1}_{\left\{Y\left(T_{n}\right) \in D_{r}\right\}} \mid \mathcal{F}_{n}\right)-\mathbb{E}^{\mathbf{x}}\left(T_{n+1}-T_{n} \mid \mathcal{F}_{n}\right) \\
& =\frac{1}{c_{0}} \mathbf{1}_{\left\{Y\left(T_{n}\right) \notin D_{r}\right\}} \mathbb{P}^{\mathbf{x}}\left(Y\left(T_{n+1}\right) \in D_{r} \mid \mathcal{F}_{n}\right)-\mathbb{E}^{\mathbf{x}}\left(T_{n+1}-T_{n} \mid \mathcal{F}_{n}\right) .
\end{aligned}
$$

We have on the event $\left\{Y\left(T_{n}\right) \notin D_{r}\right\}$,

$$
\mathbb{E}^{\mathbf{x}}\left(M_{n+1}-M_{n} \mid \mathcal{F}_{n}\right) \geq \frac{1}{c_{0}} \mathbb{P}^{X^{k}\left(T_{n}\right)}\left(T\left(D_{r}\right)<T_{\partial D}\right)-\mathbb{E}^{X^{k}\left(T_{n}\right)} T_{\partial D} \geq 0,
$$

by (6.1). On the event $\left\{Y\left(T_{n}\right) \in D_{r}\right\}$, we have $T_{n+1}=T_{n}, Y_{T_{n+1}} \in D_{r}$, and so

$$
\mathbb{E}^{\mathbf{x}}\left(M_{n+1}-M_{n} \mid \mathcal{F}_{n}\right)=0 .
$$

Combining the last two formulas, we conclude that $\left\{M_{n}\right\}$ is a submartingale with respect to $\left\{\mathcal{F}_{n}\right\}$.

Define

$$
S=\inf \left\{j: T_{j} \geq 1\right\} \wedge \inf \left\{j: Y_{T_{j}} \in D_{r}\right\} .
$$

Fix an $\mathbf{x} \in D^{N}$ and consider two cases. First, we may have

$$
\mathbb{P}^{\mathbf{x}}\left(S=\inf \left\{j: Y_{T_{j}} \in D_{r}\right\}\right) \geq 1 / 2 .
$$

In this case,

$$
\mathbb{P}^{\mathbf{x}}\left(T_{D_{r}}^{X^{k}} \leq 1\right) \geq 1 / 2
$$

The second case is when

$$
\mathbb{P}^{\mathbf{x}}\left(S=\inf \left\{j: Y_{T_{j}} \in D_{r}\right\}\right)<1 / 2 .
$$

In this case, $\mathbb{P}^{\mathbf{x}}(S \geq 1) \geq 1 / 2$, so $\mathbb{E}^{\mathbf{x}} T_{S} \geq 1 / 2$. The submartingale $M_{n}$ is bounded above by $1 / c_{0}$ so we can apply the optional stopping theorem to obtain

$$
\mathbb{E}^{\mathbf{x}} M_{S} \geq \mathbb{E}^{\mathbf{x}} M_{0}=0 \text {. }
$$

Hence

$$
\mathbb{P}^{\mathbf{x}}\left(Y_{T_{S}} \in D_{r}\right) \geq c_{0} \mathbb{E}^{\mathbf{x}} T_{S} \geq c_{0} / 2
$$

We will show that for some $t_{0}$,

$$
\mathbb{P}^{\mathrm{x}}\left(T_{D_{r}}^{X^{k}} \leq t_{0}\right) \geq c_{0} / 4
$$


If $T_{S}>s_{0}$ for some $s_{0}>1$ then $X_{t}^{k}$ must not hit $D_{r} \cup \partial D$ for $t \in\left(1, s_{0}\right)$. The probability of this event is bounded above by the probability of the event that Brownian motion starting from $X_{1}^{k}$ will not leave the ball $B\left(X_{1}^{k}, 2 \operatorname{diam}(D)\right)$ for $s_{0}-1$ units of time. The last probability is $c_{1}<1$, depending on $s_{0}>1$, but not depending on $X_{1}^{k}$. By the Markov property,

$$
\sup _{\mathbf{x} \in D^{N}} \mathbb{P}^{\mathbf{x}}\left(T_{S}>s_{0}\right) \leq c_{1}<1 .
$$

Applying the Markov property repeatedly at times $s_{0}, 2 s_{0}, \ldots$, we obtain for any $\mathbf{x} \in D^{N}$,

$$
\mathbb{P}^{\mathbf{x}}\left(T_{S}>n s_{0}\right) \leq c_{1}^{n} .
$$

We choose $n$ so large that $c_{1}^{n} \leq c_{0} / 4$ and let $t_{0}=n s_{0}$. Then for $\mathbf{x} \in D^{N}$,

$$
\mathbb{P}^{\mathbf{x}}\left(T_{S}>t_{0}\right) \leq c_{0} / 4 .
$$

We use (6.3) and (6.5) to see that

$$
\begin{aligned}
c_{0} / 2 & \leq \mathbb{P}^{\mathbf{x}}\left(Y_{T_{S}} \in D_{r}\right) \\
& =\mathbb{P}^{\mathbf{x}}\left(Y_{T_{S}} \in D_{r}, T_{S}>t_{0}\right)+\mathbb{P}^{\mathbf{x}}\left(Y_{T_{S}} \in D_{r}, T_{S} \leq t_{0}\right) \\
& \leq \mathbb{P}^{\mathbf{x}}\left(T_{S}>t_{0}\right)+\mathbb{P}^{\mathbf{x}}\left(T_{D_{r}}^{X^{k}} \leq t_{0}\right) \\
& \leq c_{0} / 4+\mathbb{P}^{\mathbf{x}}\left(T_{D_{r}}^{X^{k}} \leq t_{0}\right) .
\end{aligned}
$$

This implies (6.4). We combine the two cases, that is, (6.2) and (6.4), to see that for some $t_{1}<\infty$ and $c_{2}$, for all $\mathbf{x} \in D^{N}$,

$$
\mathbb{P}^{\mathrm{x}}\left(T_{D_{r}}^{X^{k}} \leq t_{1}\right) \geq c_{2} .
$$

Let $r_{1}$ be such that $0<r<r_{1}$ and $\operatorname{Int} D_{r_{1}} \neq \emptyset$. Let $t_{2}$ and $c_{3}$ be such that (6.6) holds with $r_{1}, t_{2}$ and $c_{3}$ in place of $r, t_{1}$ and $c_{2}$, i.e.,

$$
\mathbb{P}^{\mathrm{x}}\left(T_{D_{r_{1}}}^{X^{k}} \leq t_{2}\right) \geq c_{3} .
$$

Let $r_{2}=\left(r_{1}-r\right) / 2$ and $p_{1}=\mathbb{P}^{0}\left(T_{\partial B\left(0, r_{2}\right)} \geq t_{2}\right)>0$. By translation invariance of Brownian motion, $p_{1}=\mathbb{P}^{y}\left(T_{\partial B\left(y, r_{2}\right)} \geq t_{2}\right)$ for every $y$. If the process $X^{k}$ hits $D_{r_{1}}$ before time $t_{2}$ and then stays in the ball $B\left(X^{k}\left(T_{D_{r_{1}}}^{X^{k}}\right), r_{2}\right)$ for at least $t_{2}$ units of time then $X^{k}$ will be inside $D_{r}$ at time $t_{2}$. By the strong Markov property applied at the stopping time $T_{D_{r_{1}}}^{X^{k}}$, we obtain, using (6.7), for all $\mathrm{x} \in D^{N}$,

$$
\mathbb{P}^{\mathbf{x}}\left(X_{t_{2}}^{k} \in D_{r}\right) \geq p_{1} \mathbb{P}^{\mathbf{x}}\left(T_{D_{r_{1}}}^{X^{k}} \leq t_{2}\right) \geq p_{1} c_{3}>0 .
$$

This completes the proof of part (i) of the lemma. 
(ii) Recall that $r>0$ is fixed and such that $\operatorname{Int} D_{r} \neq \emptyset$. Let $r_{3}$ and $r_{4}$ be such that $0<r<r_{3}<r_{4}$ and $\operatorname{Int} D_{r_{4}} \neq \emptyset$. Let $r_{5}=\min \left(r_{3}-r, r_{4}-r_{3}\right) / 2$. We choose $t_{3}$ and $c_{4}$ so that (6.8) can be applied with $r_{4}$ in place of $r$,

$$
\mathbb{P}^{\mathbf{x}}\left(X_{t_{3}}^{k} \in D_{r_{4}}\right) \geq c_{4}>0 .
$$

Let $p_{2}=\inf _{y \in D} \mathbb{P}^{y}\left(T_{\partial D} \leq t_{3}\right)$ and note that $p_{2}>0$. Let $p_{3}=\mathbb{P}^{y}\left(T_{\partial B\left(y, r_{5}\right)} \geq 2 t_{3}\right)>0$ and note that $p_{3}$ does not depend on $y$.

Let $A$ be the intersection of the following events.

(a) The process $X^{1}$ is in $D_{r_{4}}$ at time $t_{3}$, and it stays in $B\left(X_{t_{3}}^{1}, r_{5}\right)$ for all $t \in\left[t_{3}, 3 t_{3}\right]$.

(b) For every $j=2, \ldots, N$, the process $X^{j}$ jumps at a time $s_{j} \in\left[t_{3}, 2 t_{3}\right]$ to $X_{s_{j}}^{1}$, and then stays in the ball $B\left(X_{s_{j}}^{j}, r_{5}\right)=B\left(X_{s_{j}}^{1}, r_{5}\right)$ for all $t \in\left[s_{j}, s_{j}+2 t_{3}\right]$.

By the strong Markov property and the definition of the process $\mathbf{X}$, the probability of $A$ is bounded below by $c_{5}=c_{4} p_{3}\left(p_{2}(1 /(N-1)) p_{3}\right)^{N-1}$. If $A$ occurs then $\mathbf{X}_{3 t_{3}} \in D_{r}^{N}$. Hence, for every $\mathbf{x} \in D^{N}$,

$$
\mathbb{P}^{\mathbf{x}}\left(\mathbf{X}_{3 t_{3}} \in D_{r}^{N}\right) \geq c_{5}>0
$$

This proves part (ii) of the lemma.

\section{Stationary Distribution FOR THE PARTICLE SYSTEM}

The two theorems proved in this section generalize the analogous results in [10], where the proofs were given only for domains satisfying the internal ball condition.

Theorem 7.1. Suppose that $D \subset \mathbb{R}^{d}$ is a bounded Lipschitz domain with the Lipschitz constant $L<c(N, d)$, where $c(N, d)$ is as in Theorem 5.1. Then there exists a unique stationary probability distribution $\mathcal{M}^{N}$ for $\mathbf{X}_{t}$. The process $\mathbf{X}_{t}$ converges to its stationary distribution exponentially fast, i.e., there exists $\lambda>0$ such that for every $A \subset D^{N}$,

$$
\lim _{t \rightarrow \infty} \mathrm{e}^{\lambda t} \sup _{\mathbf{x} \in D^{N}}\left|\mathbb{P}^{\mathbf{x}}\left(\mathbf{X}_{t} \in A\right)-\mathcal{M}^{N}(A)\right|=0 .
$$

Proof. We have shown in Lemma 6.1 (ii) that for any $r>0$, with probability higher than $p_{0}=p_{0}(r)>0$, the process $\mathbf{X}_{t}$ can reach the compact set $D_{r}^{N}$ within $t_{0}>0$ units of time. This and the strong Markov property applied at times $2 t_{0}, 4 t_{0}, 6 t_{0}, \ldots$ show that the hitting time of $D_{r}^{N}$ is stochastically bounded by an exponential random variable with the expectation independent of the starting point of $\mathbf{X}_{t}$. Since the transition densities $p_{t}^{\mathbf{X}}(\mathbf{x}, \mathbf{y})$ for $\mathbf{X}_{t}$ are bounded below by the densities for the Brownian motion killed at the exit time from $D^{N}$, we see that $p_{t}^{\mathbf{X}}(\mathbf{x}, \mathbf{y})>c_{1}>0$ for $\mathbf{x}, \mathbf{y} \in D_{r}^{N}$. Fix arbitrarily small $s>0$ and consider the "skeleton" $\left\{\mathbf{X}_{n s}\right\}_{n \geq 0}$. The properties listed in this paragraph imply that the skeleton has a stationary probability distribution and that it converges to that distribution exponentially 
fast, i.e., (7.1) holds for the skeleton, by Theorem 2.1 in [15] or Theorem 16.0.2 (ii) and (vi) of [21]. See the proof of Proposition 1.2 in [9] for an argument showing how to pass from the the statement of uniform ergodicity for the skeleton to the analogous statement for the continuous process $t \rightarrow \mathbf{X}_{t}$. We sketch this argument here. Take any $\varepsilon>0$ and find $t_{1}=n_{1} s$ such that

$$
\mathrm{e}^{\lambda t} \sup _{\mathbf{x} \in D^{N}}\left|\mathbb{P}^{\mathbf{x}}\left(\mathbf{X}_{t} \in A\right)-\mathcal{M}^{N}(A)\right| \leq \varepsilon
$$

holds for $t \geq t_{1}$ of the form $t=n s$. Consider an arbitrary $t_{2}>t_{1}$, not necessarily of the form $n s$. Let $m$ be the integer part of $t_{2} / s$ and let $u=t_{2}-m s$. Note that $m \geq n_{1}$. Since (7.2) holds for $t=m s$, the semigroup property applied at time $u$ shows that (7.2) holds also at time $t_{2}$.

Theorem 7.2. Suppose that $D$ is a bounded Lipschitz domain with the Lipschitz constant $L<\frac{1}{\sqrt{d-1}}$. For $N \geq N_{0}$ (see Remark 5.2) let $\mathcal{X}_{\mathcal{M}}^{N}$ be the stationary empirical measure. Let $\varphi$ be the first eigenfunction for Laplacian in D with the Dirichlet boundary conditions, normalized so that $\int_{D} \varphi=1$. Then the sequence of random measures $\mathcal{X}_{\mathcal{M}}^{N}, N \geq N_{0}$, converges as $N \rightarrow \infty$ to the (non-random) measure with the density $\varphi$, in the sense of weak convergence of random measures.

Proof. Recall processes $Y^{j}$ defined in the proof of Theorem 5.1. By construction, we have $\operatorname{dist}\left(Y_{t}^{j}, \partial D\right) \leq \operatorname{dist}\left(X_{t}^{j}, \partial D\right)$, for all $j$ and $t$.

It is elementary to see that the process $Z$ constructed in Section 4 has the property that

$$
\lim _{r \downarrow 0} \limsup _{t \rightarrow \infty} \frac{1}{t} \int_{0}^{t} \mathbf{1}_{\left\{\operatorname{dist}\left(Z_{s}, \partial D\right) \leq r\right\}} d s=0 \text {, a.s. }
$$

In view of the construction of $Y^{j}$ from independent copies of $Z$, we also have, for every $j$,

$$
\lim _{r \downarrow 0} \limsup _{t \rightarrow \infty} \frac{1}{t} \int_{0}^{t} \mathbf{1}_{\left\{\operatorname{dist}\left(Y_{s}^{j}, \partial D\right) \leq r\right\}} d s=0 \text {, a.s. }
$$

Hence, for every $j$,

$$
\lim _{r \downarrow 0} \limsup _{t \rightarrow \infty} \frac{1}{t} \int_{0}^{t} \mathbf{1}_{\left\{\operatorname{dist}\left(X_{s}^{j}, \partial D\right) \leq r\right\}} d s=0 \text {, a.s. }
$$

This implies that for every $p_{1}>0$, one can find $r>0$ so small that if $\mathbf{X}$ has the stationary measure $\mathcal{M}^{N}$ then for every $t, \mathbb{P}\left(X_{t}^{j} \notin D_{r}\right) \leq p_{1}$. It follows that for any $N$, the mean measure $E \mathcal{X}_{\mathcal{M}}^{N}$ of the compact set $D_{r}$ is not less than $1-p_{1}$. Hence, the mean measures $E \mathcal{X}_{\mathcal{M}}^{N}$ are tight in $D$. Lemma 3.2.7, p. 32, of [13] implies that the sequence of random measures $\mathcal{X}_{\mathcal{M}}^{N}$ is tight and so it contains a convergent subsequence. 
One can complete the proof of the claim that the random measures $\mathcal{X}_{\mathcal{M}}^{N}$ converge as $N \rightarrow \infty$ to the measure with the density $\varphi$ exactly as in the proof of Theorem 1.4 in [10], starting on line 9 of page 699 .

\section{Polyhedral DOMAins}

In this section we show that the Lipschitz constant $c(N, d)$ in Theorem 5.1 is not sharp, that is, $\tau_{\infty}=\infty$, a.s., in some Lipschtz domains with arbitrarily large Lipschitz constant. Specifically, we will demonstrate the existence of the two particle process for all times in arbitrary polyhedral domains. Unfortunately, our method cannot be easily adapted to the multiparticle case, so we leave this generalization as an open problem.

Definition 8.1. We say an open set $D \subset \mathbb{R}^{d}$ is a polyhedral domain if there exist simplicial complexes $\mathcal{K} \supset \partial \mathcal{K}$ such that $\bar{D}=|\mathcal{K}|$ and $\partial D=|\partial \mathcal{K}|$.

For the remainder of this section we will assume that $D=\operatorname{Int}|\mathcal{K}|$ is a polyhedral domain. Let $\mathbf{X}_{t}=\left(X_{t}^{1}, X_{t}^{2}\right)$ be a Fleming-Viot process in $D$ and define jump times $\tau_{i}$ as before. We will show:

Theorem 8.2. If $D$ is a polyhedral domain and $\mathbf{X}_{t}=\left(X_{t}^{1}, X_{t}^{2}\right)$ is a Fleming-Viot process with jump times $\tau_{i}$ then $\tau_{i} \rightarrow \infty$ as $i \rightarrow \infty$ almost surely.

As $\mathbf{X}_{t}$ is a CÀdLÀg process we have $X_{\tau_{i}}^{1}=X_{\tau_{i}}^{2}$ for each $i \in \mathbb{N}$, so we may define a sequence of jump points $\xi_{i}=X_{\tau_{i}}^{1}=X_{\tau_{i}}^{2}$. Since $\bar{D}$ is compact, $\xi_{i}$ has at least one limit point in $\bar{D}$. To prove Theorem 8.2 we will examine the behavior of $\mathbf{X}_{t}$ when both particles are close to a limit point of $\xi_{i}$ and, assuming that $\tau_{\infty}<\infty$, arrive at a contradiction.

First we will show that if $t \in\left[\tau_{i}, \tau_{i+1}\right)$ then $\mathbf{X}_{t}$ cannot stray too far from $\left(\xi_{i}, \xi_{i}\right)$.

Lemma 8.3. Set $V_{t}^{1}=\left\|X_{t}^{1}-\xi_{i}\right\|, V_{t}^{2}=\left\|X_{t}^{2}-\xi_{i}\right\|$ for $t \in\left[\tau_{i}, \tau_{i+1}\right)$. If $\tau_{\infty}<\infty$ then $V_{t}^{1} \rightarrow 0$ and $V_{t}^{2} \rightarrow 0$ as $t \rightarrow \tau_{\infty}$.

Proof. It suffices to consider only $V_{t}^{1}$. Notice that $V_{t}^{1}$ is a $d$-dimensional Bessel process (Bes $(d)$, for short), reset to 0 at each $\tau_{i}$. So setting $\Delta V_{i}^{1}=V_{\tau_{i}^{-}}^{1}$ we may extract a Brownian motion

$$
W_{t}=V_{t}^{1}+\sum_{\left\{i \in \mathbb{N}: \tau_{i} \leq t\right\}} \Delta V_{i}^{1}-\int_{0}^{t} \frac{d-1}{2 V_{t}^{1}} d t .
$$

Consider $\varepsilon>0$. We will count the number of upcrossings of the interval $\left[\frac{\varepsilon}{2}, \varepsilon\right]$ within a short time interval $[t, t+\delta]$, where $\delta=\varepsilon^{2} /(4(d-1))$. Consider times $t<s^{\prime}<s<t+\delta$ where $V_{s}^{1} \geq \varepsilon$ and $s^{\prime}=\sup \left\{\tilde{s}<s: V_{\tilde{s}}^{1}=\frac{\varepsilon}{2}\right\}$. Notice as $V^{1}$ only jumps downwards there is 
no $i \in \mathbb{N}$ such that $s^{\prime}<\tau_{i} \leq s$. We have

$$
\begin{aligned}
W_{s}-W_{s^{\prime}} & =V_{s}^{1}-V_{s^{\prime}}^{1}-\int_{s^{\prime}}^{s} \frac{d-1}{2 V_{t}^{1}} d t \\
& \geq \frac{\varepsilon}{2}-\left(s-s^{\prime}\right) \frac{d-1}{\varepsilon} \\
& \geq \frac{\varepsilon}{2}-\frac{\varepsilon^{2}}{4(d-1)} \frac{d-1}{\varepsilon}=\frac{\varepsilon}{4} .
\end{aligned}
$$

So on a short time interval, each upcrossing of $\left[\frac{\varepsilon}{2}, \varepsilon\right]$ by $V^{1}$ corresponds to an oscillation of $\frac{\varepsilon}{4}$ by $W$. As $W$ is a Brownian motion, with probability $1, V^{1}$ makes only finitely many upcrossings of $\left[\frac{\varepsilon}{2}, \varepsilon\right]$ in a given time interval $[t, t+\delta]$. If $\tau_{\infty}<\infty$, we may find $n \in \mathbb{N}$ with $\tau_{n} \geq \tau_{\infty}-\delta$. So if $V_{t}^{1}>\varepsilon$ for some $\tau_{n}<\tau_{i}<t<\tau_{i+1}$ then as $V^{1}$ is reset to 0 at $\tau_{i}$ there must be an upcrossing of $\left[\frac{\varepsilon}{2}, \varepsilon\right]$ in the interval $\left[\tau_{i}, \tau_{i+1}\right) \subset\left[\tau_{n}, \tau_{n}+\delta\right]$. So $V_{t}^{1}>\varepsilon$ in only finitely many intervals $\left[\tau_{i}, \tau_{i+1}\right)$ and, as $\varepsilon$ is arbitrary, $V_{t}^{1} \rightarrow 0$ as $t \rightarrow \tau_{\infty}$.

Corollary 8.4. If $\tau_{\infty}<\infty$ then the sequence $\xi_{i}$ has no limit point $\xi_{\infty} \in D$.

Proof. Fix $x \in D$. As $D$ is open, there exists some $\varepsilon$ with $B(x, 2 \varepsilon) \subset D$. If $\xi_{i} \in B(x, \varepsilon)$ then, as both particles follow continuous paths until one exits $D$, we must have $V_{t}^{1} \vee V_{t}^{2}>\varepsilon$ for some $t \in\left[\tau_{i}, \tau_{i+1}\right)$. So if $V_{t}^{1}, V_{t}^{2} \rightarrow 0$ as $t \rightarrow \tau_{\infty}$ then $\xi_{i} \in B(x, \varepsilon)$ for only finitely many $i$. As $x$ is arbitrary we see that so long as $V_{t}^{1}, V_{t}^{2} \rightarrow 0$ as $t \rightarrow \tau_{\infty}, \xi_{i}$ can have no limit point in $D$.

Corollary 8.4 is similar to a result in [19] (Step 1 of Theorem 7). In that paper, a system consisting of an arbitrary number of particles is considered, but the boundary $\partial D$ is assumed to be smooth.

It is convenient at this point to introduce some notation that will allow us to consider the behavior of $\mathbf{X}_{t}$ when it is close to the boundary of a simplicial complex. Let $\sigma$ be a $k$-simplex with vertices $\left\{v_{0}, \ldots, v_{k}\right\}$, that is

$$
\sigma=\left\{\sum_{i=0}^{k} \lambda_{i} v_{i}: \lambda_{0}, \ldots, \lambda_{k} \geq 0, \sum_{i=0}^{k} \lambda_{i}=1\right\}
$$

Then define the interior of $\sigma$

$$
\stackrel{\circ}{\sigma}=\left\{\sum_{i=0}^{k} \lambda_{i} v_{i}: \lambda_{0}, \ldots, \lambda_{k}>0, \sum_{i=0}^{k} \lambda_{i}=1\right\}
$$

and the span of $\sigma$ to be the subspace

$$
\mathcal{S}_{\sigma}=\left\{\sum_{i=0}^{k} \lambda_{i} v_{i}: \sum_{i=0}^{k} \lambda_{i}=0\right\} .
$$


For two simplices $\sigma_{1}, \sigma_{2} \in \mathcal{K}$ we write $\sigma_{1} \leq \sigma_{2}$ if $\sigma_{1}$ is a face of $\sigma_{2}$ and $\sigma_{1}<\sigma_{2}$ if $\sigma_{1}$ is a proper face of $\sigma_{2}$. We name the star of a simplex $\sigma$ to be the set

$$
S t(\sigma)=\left\{\sigma_{1} \in \mathcal{K}: \sigma_{1} \geq \sigma\right\}
$$

and define the neighborhood of $\sigma$ as

$$
\mathcal{N}(\sigma)=\left\{x \in \bar{D}: x \in \stackrel{\circ}{\sigma}_{1} \text { for some } \sigma_{1} \geq \sigma\right\} .
$$

Given simplices $\sigma \leq \sigma_{1}$ name the vertices of $\sigma$ and $\sigma_{1},\left\{v_{0}, \ldots, v_{k}\right\}$ and $\left\{v_{0}, \ldots, v_{n}\right\}$ respectively. Define the wedges

$$
\begin{aligned}
\mathcal{W}\left(\sigma, \sigma_{1}\right) & =\left\{\sum_{i=0}^{n} \lambda_{i} v_{i}: \lambda_{k+1}, \ldots, \lambda_{n}>0, \sum_{i=0}^{n} \lambda_{i}=1\right\}, \\
\mathcal{W}(\sigma) & =\bigcup_{\sigma_{1} \in S t(\sigma)} \mathcal{W}\left(\sigma, \sigma_{1}\right) .
\end{aligned}
$$

Notice that $\mathcal{N}(\sigma) \subset \overline{\mathcal{W}(\sigma)}$ and that $\mathcal{N}(\sigma)$ is open with respect to the subspace topology of $\bar{D}$. Notice also that $\mathcal{W}(\sigma)$ is a product space

$$
\mathcal{W}(\sigma)=\mathcal{C}(\sigma) \times \mathcal{S}_{\sigma}
$$

where the cone $\mathcal{C}(\sigma)$ is the projection of $\mathcal{W}(\sigma)$ onto $\mathcal{S}_{\sigma}^{\perp}$.

Now, consider $\sigma \in \partial \mathcal{K}$ and suppose there exists a subsequence $\xi_{i_{n}} \rightarrow \xi_{\infty} \in \stackrel{\circ}{\sigma}$. As $\xi_{\infty} \in \stackrel{\circ}{\sigma} \subset$ $\mathcal{N}(\sigma)$ and $\mathcal{N}(\sigma)$ is open in $\bar{D}$ we may assume without loss of generality that $\xi_{i_{n}} \in \mathcal{N}(\sigma)$ for each $n$. So consider $\mathbf{X}_{t}$ started at $\left(\xi_{i_{n}}, \xi_{i_{n}}\right)$ at time $\tau_{i_{n}}$ and stopped at the first time $T>\tau_{i_{n}}$ where one of $X_{t}^{1}, X_{t}^{2}$ exits $\mathcal{N}(\sigma)$. Of course, as $\mathcal{N}(\sigma) \subset \mathcal{W}(\sigma) \cap \bar{D}$, this has the same distribution as a Fleming-Viot process in $\mathcal{W}(\sigma)$ started and stopped in the same way.

So, let $\mathbb{P}_{\sigma}^{x}$ and $\mathbb{E}_{\sigma}^{x}$ be the probability measure and expectation operator associated with a Fleming-Viot process in $\mathcal{W}(\sigma)$ started at $\mathbf{X}_{0}=(x, x)$. The $\mathcal{S}_{\sigma}$ and $\mathcal{S}_{\sigma}^{\perp}$ components are not quite independent as they have the same jumps, but $\mathbb{P}_{\sigma}$ allows a partial factorization as follows.

Lemma 8.5. If $\mathbf{X}_{t}$ is a Fleming-Viot process in $\mathcal{W}(\sigma)$ then there is a well defined decomposition $\mathbf{X}_{t}=\mathbf{Y}_{t}+\mathbf{Z}_{t}$ with $\mathbf{Y}_{t}=\left(Y_{t}^{1}, Y_{t}^{2}\right) \in \mathcal{C}(\sigma)^{2}, \mathbf{Z}_{t}=\left(Z_{t}^{1}, Z_{t}^{2}\right) \in \mathcal{S}_{\sigma}^{2}$ with the following properties

- $\mathbf{Y}_{t}$ is a Fleming-Viot process in $\mathcal{C}(\sigma)$;

- there exists a Brownian motion $\tilde{Z}_{t}$ in $\mathcal{S}_{\sigma}$ (not adapted to the filtration of $\mathbf{X}_{t}$ ), independent of $\mathbf{Y}_{t}$, such that for each $i \in \mathbb{N}$ we have $\tilde{Z}_{\tau_{i}}=\zeta_{i}$ with $\zeta_{i}=Z_{\tau_{i}}^{1}=Z_{\tau_{i}}^{2}$.

Proof. Obviously, as $\mathcal{C}(\sigma) \subset \mathcal{S}_{\sigma}^{\perp}$, the factorization $\mathbf{X}_{t}=\mathbf{Y}_{t}+\mathbf{Z}_{t}$ is unique. Further, on each interval $\left[\tau_{i}, \tau_{i+1}\right)$, the processes $Y_{t}^{1}, Y_{t}^{2}, Z_{t}^{1}$ and $Z_{t}^{2}$ evolve as independent Brownian motions 
on $\mathcal{S}_{\sigma}^{\perp}$ and $\mathcal{S}_{\sigma}$ respectively. So as $\mathcal{S}_{\sigma}$ is a subspace and has no boundary, $X_{t}^{j}$ jumps when and only when $Y_{t}^{j}$ hits $\partial \mathcal{C}(\sigma)$, and so $\mathbf{Y}_{t}$ is indeed a Fleming-Viot process on $\mathcal{C}(\sigma)$.

Now for each $i \in \mathbb{N}$ only one of $X_{t}^{1}, X_{t}^{2}$ has a discontinuity at $\tau_{i+1}$, so there is a well defined sequence of random variables $J_{i} \in\{1,2\}$ such that $X_{t}^{J_{i}}$ is continuous on the closed interval $\left[\tau_{i}, \tau_{i+1}\right]$ and we may define a continuous process

$$
\tilde{Z}_{t}=Z_{t}^{J_{i}}, \quad t \in\left[\tau_{i}, \tau_{i+1}\right] .
$$

Then $\tilde{Z}_{\tau_{i}}=\zeta_{i}$ for every $i$ and it remains to show that $\tilde{Z}_{t}$ is a Brownian motion independent of $\mathbf{Y}_{t}$. Of course $\tilde{Z}_{t}$ is only defined up to $\tau_{\infty}$. But we may continue $\tilde{Z}_{t}$ after $\tau_{\infty}$ with an independent Brownian motion if necessary.

Now as $\tilde{Z}_{t}$ follows either $Z_{t}^{1}$ or $Z_{t}^{2}$ then the quadratic variation $\langle\tilde{Z}\rangle_{t}=t \mathrm{I}$ and, by Lévy's characterization, we need only check that $\tilde{Z}_{t}$ is a martingale with respect to its own natural filtration and is independent of $\mathbf{Y}_{t}$. Furthermore, although $\tilde{Z}_{t}$ is not adapted to $\mathbf{X}_{t}$, for each $\tau_{i}$, the path $\left.\tilde{Z}\right|_{\left[0, \tau_{i}\right]}$ is measurable with respect to $\left.\mathbf{X}\right|_{\left[0, \tau_{i}\right]}$. Therefore, by the strong Markov property, it is sufficient to consider only intervals $\left[\tau_{i}, \tau_{i+1}\right)$.

In fact it suffices to consider only the first time interval $\left[0, \tau_{1}\right)$. Let $\mathbf{X}_{t}$ be a Fleming-Viot process started at $\xi_{0} \in \mathcal{W}(\sigma)$ and stopped at $\tau_{1}$. Then the left limit process is a pair of independent Brownian motions stopped at $\tau=\tau_{1}^{-}$. Set $J=J_{0}$ and we have $\xi_{1}=X_{\tau}^{J} \in \mathcal{W}(\sigma)$ and $X_{\tau}^{3-J} \in \partial \mathcal{W}(\sigma)$.

So set $\mathbf{X}_{t}=\mathbf{Y}_{t}+\mathbf{Z}_{t}$ as in the statement of the lemma and let $\mathcal{F}_{t}^{\mathbf{Y}}, \mathcal{F}_{t}^{\mathbf{Z}}$ and $\mathcal{F}_{t}^{\tilde{Z}}$ be the natural filtrations of $\mathbf{Y}, \mathbf{Z}$ and $\tilde{Z}$ respectively. Set $\zeta_{0}=Z_{0}^{1}, \zeta_{1}=Z_{\tau}^{J}$ to be the $\mathcal{F}_{\tau}^{\mathbf{X}}$ measurable $\mathbf{Z}$-components of $\xi_{0}$ and $\xi_{1}$, respectively. Thus, $\tau$ is a stopping time of $\mathcal{F}_{t}^{\mathbf{Y}}$ and $J$ is measurable with respect to $\mathcal{F}_{\tau}^{\mathbf{Y}}$. Now crucially $\mathbf{Y}$ and $\mathbf{Z}$ are independent processes so for $t<\tau$ we have

$$
\mathbb{E}_{\sigma}^{\xi_{0}}\left(\zeta_{1} \mid \mathcal{F}_{\tau}^{\mathbf{Y}} \vee \mathcal{F}_{t}^{\mathbf{Z}}\right)=\mathbb{E}_{\sigma}^{\xi_{0}}\left(Z_{\tau}^{J} \mid \mathcal{F}_{\tau}^{\mathbf{Y}} \vee \mathcal{F}_{t}^{\mathbf{Z}}\right)=\tilde{Z}_{t}
$$

Thus $\tilde{Z}$ is a martingale, and hence a Brownian motion, with respect to the filtration $\mathcal{G}_{t}=\mathcal{F}_{\tau}^{\mathbf{Y}} \vee \mathcal{F}_{t}^{\mathbf{Z}}$. Therefore $\tilde{Z}$ is independent of $\mathcal{F}_{\tau}^{\mathbf{Y}} \subset \mathcal{G}_{0}$ and is a Brownian motion with respect to its own natural filtration $\mathcal{F}_{t}^{\tilde{Z}} \subset \mathcal{G}_{t}$.

Now $\mathbf{Y}_{t}$ is a process in a cone and if $\xi_{i}$ converges to some point in $\stackrel{\circ}{\sigma}$ then $\mathbf{Y}_{t}$ must converge to the apex of $\mathcal{C}(\sigma)$. Our next step is to show that this cannot be the case.

Lemma 8.6. If $\mathbf{Y}_{t}$ is a Fleming-Viot process in a cone $C \subset \mathbb{R}^{d}$ then, with probability one, $\mathbf{Y}_{t}$ does not converge to $(\underline{0}, \underline{0})$. 
To prove this we will need to consider the angular components, $\Phi_{t}^{j}=\frac{Y_{t}^{j}}{\left\|Y_{t}^{j}\right\|}$, of $\mathbf{Y}$. We will recall briefly some facts about spherical Brownian motion. We will omit details, which can be found in [23, Chapter 8], particularly Example 8.5.8.

Let $B_{t}$ be a Brownian motion on $\mathbb{R}^{d}$, let the unit sphere be denoted

$$
\mathbb{S}^{d-1}=\left\{x \in \mathbb{R}^{d}:\|x\|=1\right\},
$$

and define the map $\phi: \mathbb{R}^{d} \backslash\{\underline{0}\} \rightarrow \mathbb{S}^{d-1}$ by $\phi(x)=\frac{x}{\|x\|}$.

Now let $\Phi_{t}=\phi\left(B_{t}\right)$. Applying Ito's formula,

$$
d \Phi_{t}=\frac{1}{\left\|B_{t}\right\|}\left(I-\Phi_{t} \Phi_{t}^{\top}\right) d B_{t}-\frac{d-1}{2\left\|B_{t}\right\|^{2}} \Phi_{t} d t .
$$

Note we are interpreting $\Phi_{t}$ as a column vector so $\Phi_{t} \Phi_{t}^{\top}$ is a square matrix. Now define a differential operator $A: \mathcal{C}^{2}\left(\mathbb{S}^{d-1}, \mathbb{R}\right) \rightarrow \mathcal{C}^{0}\left(\mathbb{S}^{d-1}, \mathbb{R}\right)$ by

$$
A f(x)=\frac{1}{2}\left(\Delta f(x)-\sum_{i, j} x_{i} x_{j} \frac{\partial^{2} f}{\partial x_{i} \partial x_{j}}\right)-\frac{d-1}{2} \sum_{i} x_{i} \frac{\partial f}{\partial x_{i}} .
$$

Applying Ito's formula again, we see that $f\left(\Phi_{t}\right)-\int_{0}^{t} \frac{A f\left(\Phi_{t}\right)}{\left\|B_{t}\right\|^{2}} d t$ is a local martingale for each $f \in \mathcal{C}^{2}\left(\mathbb{S}^{d-1}, \mathbb{R}\right)$.

We may extend this to functions of two Brownian motions by defining $\mathcal{A}^{1}, \mathcal{A}^{2}$ by

$$
\begin{aligned}
& \mathcal{A}^{1} f(x, y)=A(f(\cdot, y))(x), \\
& \mathcal{A}^{2} f(x, y)=A(f(x, \cdot))(y) .
\end{aligned}
$$

Then by a similar application of Ito's formula, if $B_{t}^{1}$ and $B_{t}^{2}$ are independent Brownian motions and $\Phi_{t}^{1}=\phi\left(B_{t}^{1}\right), \Phi_{t}^{2}=\phi\left(B_{t}^{2}\right), \Phi_{t}=\left(\Phi_{t}^{1}, \Phi_{t}^{2}\right)$, then

$$
N_{t}^{f}=f\left(\Phi_{t}^{1}, \Phi_{t}^{2}\right)-\int_{0}^{t}\left(\frac{\mathcal{A}^{1} f\left(\Phi_{t}\right)}{\left\|B_{t}^{1}\right\|^{2}}+\frac{\mathcal{A}^{2} f\left(\Phi_{t}\right)}{\left\|B_{t}^{2}\right\|^{2}}\right) d t
$$

is a local martingale.

Now apply a time change to $\Phi_{t}$ as follows. If $\alpha(t)=\inf \left\{s \in \mathbb{R}^{+}: \int_{0}^{s}\left\|B_{\tilde{s}}\right\|^{-2} d \tilde{s} \geq t\right\}$, then $\Theta_{t}=\Phi_{\alpha(t)}$ is a Markov diffusion on $\mathbb{S}^{d-1}$ with generator $A$. Let $\mathbb{P}_{\mathbb{S}}^{\theta_{1}, \theta_{2}}$ and $\mathbb{E}_{\mathbb{S}}^{\theta_{1}, \theta_{2}}$ be the probability measure and expectation operator associated with two independent copies of $\Theta_{t}$ started at $\theta_{1}$ and $\theta_{2} \in \mathbb{S}^{d-1}$ respectively.

Lemma 8.7. Let $U$ be an open subset of $\mathbb{S}^{d-1}$ and set

$$
\begin{aligned}
T_{1}^{U} & =\inf \left\{t \in \mathbb{R}: \Theta_{t}^{1} \in \partial U\right\}, \\
T_{2}^{U} & =\inf \left\{t \in \mathbb{R}: \Theta_{t}^{2} \in \partial U\right\}, \\
h^{U}\left(\theta_{1}, \theta_{2}\right) & =\mathbb{P}_{\mathbb{S}}^{\theta_{1}, \theta_{2}}\left[T_{1}^{U}<T_{2}^{U}\right] .
\end{aligned}
$$


Then $h^{U} \in \mathcal{C}^{2}\left(U^{2}, \mathbb{R}\right)$ and $\mathcal{A}^{1} h^{U}=-\mathcal{A}^{2} h^{U} \geq 0$.

Proof. The process $\left(\Theta_{t}^{1}, \Theta_{t}^{2}\right)$ is a Markov diffusion with generator $\mathcal{A}^{1}+\mathcal{A}^{2}$, so by Dynkin's formula $\mathcal{A}^{1} h^{U}+\mathcal{A}^{2} h^{U}=0$ and it remains to show that $\mathcal{A}^{1} h^{U} \geq 0$.

By definition of the Markov generator

$$
\begin{aligned}
\mathcal{A}^{1} h^{U}\left(\theta_{1}, \theta_{2}\right) & =\lim _{t \rightarrow 0} \frac{1}{t}\left(\mathbb{E}_{\mathbb{S}}^{\theta_{1}, \theta_{2}}\left(h^{U}\left(\Theta_{t}^{1}, \theta_{2}\right)\right)-h^{U}\left(\theta_{1}, \theta_{2}\right)\right) \\
& =\lim _{t \rightarrow 0} \frac{1}{t}\left(\mathbb{E}_{\mathbb{S}}^{\theta_{1}, \theta_{2}}\left(\mathbb{P}_{\mathbb{S}}^{\Theta_{t}^{1}, \theta_{2}}\left[T_{1}^{U}<T_{2}^{U}\right]\right)-\mathbb{P}_{\mathbb{S}}^{\theta_{1}, \theta_{2}}\left[T_{1}^{U}<T_{2}^{U}\right]\right) .
\end{aligned}
$$

But $\mathbb{E}_{\mathbb{S}}^{\theta_{1}, \theta_{2}}\left(\mathbb{P}_{\mathbb{S}}^{\Theta_{t}^{1}, \theta_{2}}(\cdot)\right)$ is the probability measure associated with the process $\left(\Theta_{s+t}^{1}, \Theta_{s}^{2}\right)$, $s>0$, obtained by giving $\Theta_{1}$ a headstart. So we have

$$
\begin{aligned}
\mathbb{E}_{\mathbb{S}}^{\theta_{1}, \theta_{2}}\left(\mathbb{P}_{\mathbb{S}}^{\Theta_{t}^{1}, \theta_{2}}\left[T_{1}^{U}<T_{2}^{U}\right]\right) & \geq \mathbb{P}_{\mathbb{S}}^{\theta_{1}, \theta_{2}}\left[T_{1}^{U}-t<T_{2}^{U}\right]-\mathbb{P}_{\mathbb{S}}^{\theta_{1}, \theta_{2}}\left[T_{1}^{U}<t\right] \\
& \geq \mathbb{P}_{\mathbb{S}}^{\theta_{1}, \theta_{2}}\left[T_{1}^{U}<T_{2}^{U}\right]-\mathbb{P}_{\mathbb{S}}^{\theta_{1}, \theta_{2}}\left[T_{1}^{U}<t\right]
\end{aligned}
$$

and, since $\frac{1}{t} \mathbb{P}_{\mathbb{S}}^{\theta_{1}, \theta_{2}}\left(T_{1}^{U}<t\right) \rightarrow 0$ as $t \rightarrow 0$, we may pass to the limit, and we see that $\mathcal{A}^{1} h^{U}\left(\theta^{1}, \theta^{2}\right) \geq 0$.

We are ready to prove Lemma 8.6.

Proof of Lemma 8.6. Set $C=\left\{\lambda u: \lambda \in \mathbb{R}^{+}, u \in U\right\}$ for some open subset $U \subset \mathbb{S}^{d-1}$ and let $\mathbf{Y}_{t}$ be a Fleming-Viot process in $C$.

We deal first with the special case when $d=1$, in which case either $C=\mathbb{R}$ and there is nothing to prove or $C=\mathbb{R}^{+}$. If $C=\mathbb{R}^{+}$then $\mathbf{Y}_{t}$ is a 2-dimensional Brownian motion in the quarter plane with jumps $(y, 0) \mapsto(y, y)$ or $(0, y) \mapsto(y, y)$ whenever the process exits the first quadrant. As these jumps only increase $\left\|\mathbf{Y}_{t}\right\|$ then $\left\|\mathbf{Y}_{t}\right\|$ dominates a Bes (2) process and $\mathbf{Y}_{t}$ does not converge to 0 .

For $d \geq 2$ define a function

$$
\mu(x)= \begin{cases}\log \|x\|, & \text { if } d=2 \\ \frac{\|x\|^{2-d}}{2-d} & \text { if } d \geq 3\end{cases}
$$

and define processes

$$
\begin{array}{ll}
\Phi_{t}^{1}=\phi\left(Y_{t}^{1}\right), & M_{t}^{1}=\mu\left(Y_{t}^{1}\right), \\
\Phi_{t}^{2}=\phi\left(Y_{t}^{2}\right), & M_{t}^{2}=\mu\left(Y_{t}^{2}\right), \\
H_{t}=h^{U}\left(\Phi_{t}^{1}, \Phi_{t}^{2}\right), & S_{t}=M_{t}^{1}+\left(M_{t}^{2}-M_{t}^{1}\right) H_{t} .
\end{array}
$$

Now, $\mu$ is harmonic on $\mathbb{R}^{d}$ and it will be key to our argument that $M_{t}^{1}$ and $M_{t}^{2}$ are both local martingales except when $\mathbf{Y}_{t}$ jumps. We say a $\mathbf{Y}_{t}$-adatapted process $R_{t}$ is a martingale between jumps if $R_{t}-\sum_{\left\{i \in \mathbb{N}: \tau_{i} \leq t\right\}}\left(R_{\tau_{i}}-R_{\tau_{i}^{-}}\right)$is a continuous local martingale. The process 
$S_{t}$ is a convex combination of $M_{t}^{1}$ and $M_{t}^{2}$, so if both $Y_{t}^{1}$ and $Y_{t}^{2}$ converge to the origin, then $S_{t}$ converges to $-\infty$. Notice also that if $Y^{1}$ approaches $\partial C$ then $H_{t} \rightarrow 1$ and so $S_{t} \rightarrow M_{t}^{2}$. Similarly, if $Y_{t}^{2}$ approaches the boundary then $S_{t} \rightarrow M_{t}^{1}$. So $S_{t}$ is continuous.

Set

$$
N_{s}=H_{s}-\int_{0}^{s}\left(\frac{\mathcal{A}^{1} h^{U}\left(\Phi_{t}\right)}{\left\|B_{t}^{1}\right\|^{2}}+\frac{\mathcal{A}^{2} h^{U}\left(\Phi_{t}\right)}{\left\|B_{t}^{2}\right\|^{2}}\right) d t .
$$

By (8.1) $N_{t}$ is a martingale between jumps. We may check that the cross variation terms $\left\langle M^{1}, \Phi^{1}\right\rangle_{t}=\left\langle M_{t}^{2}, \Phi_{t}^{2}\right\rangle=0$ and so, as $H_{t}$ is a $\mathcal{C}^{2}$ function of $\Phi_{t}^{1}$ and $\Phi_{t}^{2}$, we have $\left\langle M^{1}, H\right\rangle_{t}=$ $\left\langle M_{t}^{2}, H\right\rangle_{t}=0$ and for $s \in\left[\tau_{i}, \tau_{i+1}\right)$ we may calculate

$$
\begin{aligned}
S_{s}=S_{\tau_{i}} & +\int_{\tau_{i}}^{s}\left(1-H_{t}\right) d M_{t}^{1}+\int_{\tau_{i}}^{s} H_{t} d M_{t}^{2}+\int_{\tau_{i}}^{s}\left(M_{t}^{2}-M_{t}^{1}\right) d H_{t} \\
=S_{\tau_{i}} & +\int_{\tau_{i}}^{s}\left(1-H_{t}\right) d M_{t}^{1}+\int_{\tau_{i}}^{s} H_{t} d M_{t}^{2}+\int_{\tau_{i}}^{s}\left(M_{t}^{2}-M_{t}^{1}\right) d N_{t} \\
& +\int_{\tau_{i}}^{s}\left(M_{t}^{2}-M_{t}^{1}\right)\left(\frac{\mathcal{A}^{1} h^{U}\left(\Phi_{t}\right)}{\left\|B_{t}^{1}\right\|^{2}}+\frac{\mathcal{A}^{2} h^{U}\left(\Phi_{t}\right)}{\left\|B_{t}^{2}\right\|^{2}}\right) d t
\end{aligned}
$$

Therefore $S_{s}-\int_{\tau_{i}}^{s}\left(M_{t}^{2}-M_{t}^{1}\right)\left(\frac{\mathcal{A}^{1} h^{U}\left(\Phi_{t}\right)}{\left\|B_{t}^{1}\right\|^{2}}+\frac{\mathcal{A}^{2} h^{U}\left(\Phi_{t}\right)}{\left\|B_{t}^{2}\right\|^{2}}\right) d t$ is a martingale between jumps.

Now from Lemma 8.7 we have $\mathcal{A}^{1} h^{U}=-\mathcal{A}^{2} h^{U} \geq 0$ and so

$$
\frac{\mathcal{A}^{1} h^{U}\left(\Phi_{t}\right)}{\left\|B_{t}^{1}\right\|^{2}}+\frac{\mathcal{A}^{2} h^{U}\left(\Phi_{t}\right)}{\left\|B_{t}^{2}\right\|^{2}}=\mathcal{A}^{1} h^{U}\left(\Phi_{t}\right)\left(\left\|B_{t}^{1}\right\|^{-2}-\left\|B_{t}^{2}\right\|^{-2}\right) \text {. }
$$

But $\mu$ is an increasing function of the norm $\|\cdot\|$, so for $\tau_{i} \leq s_{1} \leq s_{2}<\tau_{i+1}$,

$$
\int_{s_{1}}^{s_{2}}\left(M_{t}^{2}-M_{t}^{1}\right)\left(\frac{\mathcal{A}^{1} h^{U}\left(\Phi_{t}\right)}{\left\|B_{t}^{1}\right\|^{2}}+\frac{\mathcal{A}^{2} h^{U}\left(\Phi_{t}\right)}{\left\|B_{t}^{2}\right\|^{2}}\right) d t \geq 0 .
$$

Therefore $S_{t}$ is a continuous local submartingale and it cannot converge to $-\infty$. Thus $\mathbf{Y}_{t}$ does not converge to $(0,0)$.

Corollary 8.8. If $\mathbf{X}_{t}$ is a Fleming-Viot process in a polyhedral domain $D$ then with probability one the sequence of jump points $\xi_{i}$ does not converge to any $\xi_{\infty} \in \partial D$ as $i \rightarrow \infty$.

Proof. First, for $\sigma \in \partial \mathcal{K}$, let $F^{\sigma}$ be the event that $\xi_{i} \rightarrow \xi_{\infty}$ for some $\xi_{\infty} \in \stackrel{\circ}{\sigma}$ and assume without loss of generality that $\underline{0} \in \sigma$. Set

$$
F_{i}^{\sigma}=F^{\sigma} \cap\left[X_{t}^{j} \in \mathcal{N}(\sigma) ; t \geq \tau_{i}, j=1,2\right] .
$$

Then, as $\mathcal{N}(\sigma)$ is open in $\bar{D}$, from Lemma 8.3, $F_{i}^{\sigma}$ increases to $F^{\sigma}$ up to an event of probability 0. By the strong Markov property and Lemma 8.6.

$$
\mathbb{P}\left(F_{i}^{\sigma}\right)=\mathbb{P}_{\sigma}^{\xi_{i}}\left(\mathbf{Y}_{t} \rightarrow(\underline{0}, \underline{0}) \cap\left[X_{t}^{j} \in \mathcal{N}(\sigma) ; t \geq \tau_{i}, j=1,2\right]\right)=0 .
$$

So as $\partial \mathcal{K}$ is a finite set of simplices we have $\mathbb{P}\left[\exists \xi_{\infty} \in \partial D\right.$ s.t. $\xi_{i} \rightarrow \xi_{\infty}$ as $\left.i \rightarrow \infty\right]=0$. 
To complete the proof of Theorem 8.2 we consider the set

$$
L=\left\{\sigma \in \mathcal{K}: \text { there exists a subsequence } \xi_{i_{n}} \rightarrow \xi \in \stackrel{\circ}{\sigma} \text { as } n \rightarrow \infty\right\} .
$$

It is easy to check that the event $\{\sigma \in L\}$ is $\mathbf{X}$-measurable. We say $\sigma$ is a local maximum of $L$ if $L \cap S t(\sigma)=\{\sigma\}$. Of course any non-empty subset of a finite lattice contains at least one local maximum, and $L$ is non empty by compactness of $\bar{D}$. We will prove Theorem 8.2 by showing that for each $\sigma \in \mathcal{K}$ the event that $\tau_{\infty}<\infty$ and $\sigma$ is a local maximum of $L$ has probability 0 .

Proof of Theorem 8.2. Fix $\sigma \in \partial \mathcal{K}$, and note that $\mathcal{N}(\sigma) \backslash \sigma$ is non empty. We show first that if $\xi_{i}$ has a limit point in $\stackrel{\circ}{\sigma}$ and $\tau_{\infty}<\infty$, then $\xi_{i}$ has a second limit point in $\mathcal{N}(\sigma) \backslash \sigma$.

First suppose that $\sigma=\{v\}$ is a vertex of $\mathcal{K}$ and $v$ is a limit point of $\xi_{i}$. By Corollary 8.8, the sequence $\xi_{i}$ does not converge to $v$ as $i \rightarrow \infty$, so we may choose $\varepsilon>0$ such that $B(v, \varepsilon) \cap \bar{D} \subset \mathcal{N}(\sigma)$ and that $\left\|\xi_{i}-v\right\|>\varepsilon$ infinitely often. If this is the case then there are infinitely many pairs $\left(\xi_{i_{n}}, \xi_{i_{n+1}}\right)$ such that $\xi_{i_{n}} \in B(v, \varepsilon)$ and $\xi_{i_{n+1}} \notin B(v, \varepsilon)$. But from Lemma 8.3 we have $\left\|\xi_{i}-\xi_{i+1}\right\| \rightarrow 0$ as $i \rightarrow \infty$ hence $\left\|\xi_{i_{n}}-v\right\| \rightarrow \varepsilon$ as $i \rightarrow \infty$. Therefore, as $\partial B(v, \varepsilon)$ is compact, $\xi_{i}$ must have some limit point in $\partial B(v, \varepsilon) \cap \bar{D} \subset \mathcal{N}(\sigma) \backslash\{v\}$.

If $\sigma$ is a $k$-simplex for $0<k<d$ then for each $x \in \stackrel{\circ}{\sigma}$ there exists $\varepsilon>0$ such that $B(x, 2 \varepsilon) \cap \bar{D} \subset \mathcal{N}(\sigma)$. We will consider upcrossings of the interval $[\varepsilon, 2 \varepsilon]$ by $\left\|\xi_{i}-x\right\|$. Define sequences $i_{n}, j_{n} \in \mathbb{N} \cup\{\infty\}$ and $T_{n}, \eta_{n} \in \mathbb{R} \cup\{\infty\}$ by: $j_{0}=0$,

$$
\begin{aligned}
i_{n+1} & =\inf \left\{i>j_{n}: \xi_{i} \in B(x, \varepsilon)\right\}, \\
j_{n} & =\inf \left\{j>i_{n}: \xi_{j} \notin B(x, 2 \varepsilon)\right\}, \\
T_{n} & =\inf _{2}\left\{t>\tau_{i_{n}}: X_{t}^{1} \notin B(x, 2 \varepsilon) \text { or } X_{t}^{2} \notin B(x, 2 \varepsilon)\right\}, \\
\eta_{n} & =\sup _{i}\left\{\tau_{i}: \tau_{i}<T_{n}\right\} .
\end{aligned}
$$

Then we put $N=\sup \left\{n \in \mathbb{N}: j_{n}<\infty\right\}$ to be the number of upcrossings.

Note that $B(x, 2 \varepsilon) \cap \bar{D} \subset \mathcal{N}(\sigma)$ and so $\mathbf{X}_{\left(t+\tau_{i n}\right) \wedge T_{n}}$ is a Fleming-Viot process in $\mathcal{W}(\sigma)$ started at $\left(\xi_{i_{n}}, \xi_{i_{n}}\right)$ and stopped on exiting $B(x, 2 \varepsilon)$. So we may consider $\mathbb{P}_{\sigma}^{\xi_{i_{n}}}$ and factorize $\mathbf{X}_{t}=\mathbf{Y}_{t}+\mathbf{Z}_{t}$ as in Lemma 8.5. For $t \in\left[\tau_{i_{n}}, \eta_{n}\right]$, the process $\tilde{Z}_{t}$ is measurable with respect to $\left.\mathbf{X}\right|_{\left[\tau_{i_{n}}, T_{n}\right]}$ which is distributed according to $\mathbb{P}_{\sigma}^{\xi_{i_{n}}}$. Hence $\left.\tilde{Z}\right|_{\left[\tau_{i_{n}}, \eta_{n}\right]}$ is a Brownian motion in $\mathcal{S}_{\sigma}$ with respect to its own natural filtration.

Recall $\mathbf{Z}_{\tau_{i}}=\left(\zeta_{i}, \zeta_{i}\right)$ and set

$$
\tilde{V}_{t}= \begin{cases}\left\|\tilde{Z}_{t}-\zeta_{i_{n}}\right\|, & \text { if } t \in\left[\tau_{i_{n}}, \eta_{n}\right], \\ 0, & \text { otherwise. }\end{cases}
$$


Then $\tilde{V}_{t}$ is dominated by a Bes $(d)$ process reset to zero at times $\tau_{i_{n}}$. So arguing as in the proof of Lemma 8.3, if $\tau_{\infty}<\infty$ and the number of upcrossings $N=\infty$, then $\tau_{i_{n}}<\tau_{\infty}<\infty$ for each $n \in \mathbb{N}$, and $\tilde{V}_{t} \rightarrow 0$ as $\tau_{i} \rightarrow \infty$. But $\eta_{n}=\sup _{i}\left\{\tau_{i}: \tau_{i}<T_{n}\right\}$, hence $\mathbf{X}_{\eta_{n}}=\left(\xi_{k_{n}}, \xi_{k_{n}}\right)$ for some $k_{n} \in \mathbb{N}$ and either $\left\|x-X_{T_{n}}^{1}\right\|=2 \varepsilon$ or $\left\|x-X_{T_{n}}^{2}\right\|=2 \varepsilon$. So if $\tau_{\infty}<\infty$ and $N=\infty$, we must have $\left\|\xi_{k_{n}}-x\right\| \rightarrow 2 \varepsilon$ as $n \rightarrow \infty$ and so $\xi_{k_{n}}$ has a limit point $\xi_{\infty} \in \partial B(x, 2 \varepsilon) \cap \bar{D} \subset \mathcal{N}(\sigma)$. But $\tilde{V}_{t} \rightarrow 0$ as $t \rightarrow \tau_{\infty}$ with probability one, so we cannot have $\xi_{\infty} \in \sigma$ and we must have $\xi_{\infty} \in \mathcal{N}(\sigma) \backslash \sigma$.

Now let $Q^{\sigma}$ be a countably dense subset of $\stackrel{\circ}{\sigma}$ and suppose $\xi_{i}$ has some limit point $x \in \stackrel{\circ}{\sigma}$. By Corollary 8.8, $\xi_{i}$ does not converge to $x$ as $i \rightarrow \infty$ and we may choose some rational $\varepsilon>0$ such that $B(x, 3 \varepsilon) \cap \bar{D} \subset \mathcal{N}(\sigma)$ and $\left\|\xi_{i}-x\right\|>3 \varepsilon$ infinitely often. Now choose $q \in Q^{\sigma} \cap B(x, \varepsilon)$ and notice that $\left\|\xi_{i}-q\right\|$ makes infinitely many upcrossings of the interval $[\varepsilon, 2 \varepsilon]$. If $\tau_{\infty}<\infty$ then as $Q^{\sigma}$ is countable, with probability one we may find some limit point $\xi_{\infty} \in \mathcal{N}(\sigma) \backslash \sigma$.

Recall the definition of the set $L$. As $L$ is nonempty there must exist some local maximum $\sigma$. However if $\tau_{\infty}<\infty$ then, by Corollary 8.4, we have $L \subseteq \partial \mathcal{K}$. We have just shown that if $\tau_{\infty}<\infty$ then $L$ has no local maximum in $\partial \mathcal{K}$. Hence we must have $\tau_{\infty}=\infty$.

\section{REFERENCES}

1. Hiroaki Aikawa, Boundary Harnack principle and Martin boundary for a uniform domain, J. Math. Soc. Japan 53 (2001), no. 1, 119-145.

2. Amine Asselah, Pablo Ferrari, and Pablo Groisman, Quasi-stationary distributions and fleming-viot processes for finite state markov processes, (2009), Math Arxiv 0904.3039.

3. Rami Atar, Siva Athreya, and Zhen-Qing Chen, Exit time, Green function and semilinear elliptic equations, Electron. J. Probab. 14 (2009), no. 3, 50-71. MR MR2471659

4. Rodrigo Bañuelos and Robert G. Smits, Brownian motion in cones, Probab. Theory Related Fields 108 (1997), no. 3, 299-319.

5. Richard F. Bass, Probabilistic techniques in analysis, Probability and its Applications (New York), Springer-Verlag, New York, 1995.

6. Richard F. Bass and Krzysztof Burdzy, Eigenvalue expansions for Brownian motion with an application to occupation times, Electron. J. Probab. 1 (1996), no. 3, approx. 19 pp. (electronic).

7. Jean Bertoin, Lévy processes, Cambridge Tracts in Mathematics, vol. 121, Cambridge University Press, Cambridge, 1996.

8. K. Burdzy, Multidimensional Brownian excursions and potential theory, Pitman Research Notes in Mathematics Series, vol. 164, Longman Scientific \& Technical, Harlow, 1987. 
9. Krzysztof Burdzy, Zhen-Qing Chen, and Donald E. Marshall, Traps for reflected Brownian motion, Math. Z. 252 (2006), no. 1, 103-132.

10. Krzysztof Burdzy, Robert Hołyst, and Peter March, A Fleming-Viot particle representation of the Dirichlet Laplacian, Comm. Math. Phys. 214 (2000), no. 3, 679-703.

11. D. L. Burkholder, Exit times of Brownian motion, harmonic majorization, and Hardy spaces, Advances in Math. 26 (1977), no. 2, 182-205.

12. Burgess Davis, On Brownian slow points, Z. Wahrsch. Verw. Gebiete 64 (1983), no. 3, 359-367.

13. Donald A. Dawson, Infinitely divisible random measures and superprocesses, Stochastic analysis and related topics (Silivri, 1990), Progr. Probab., vol. 31, Birkhäuser Boston, Boston, MA, 1992, pp. 1-129.

14. R. Dante deBlassie, Exit times from cones in $\mathbf{R}^{n}$ of Brownian motion, Probab. Theory Related Fields 74 (1987), no. 1, 1-29.

15. D. Down, S. P. Meyn, and R. L. Tweedie, Exponential and uniform ergodicity of Markov processes, Ann. Probab. 23 (1995), no. 4, 1671-1691.

16. Ilie Grigorescu and Min Kang, Ergodic properties of multidimensional Brownian motion with rebirth, Electron. J. Probab. 12 (2007), no. 48, 1299-1322 (electronic).

17. John Hawkes, Intersections of Markov random sets, Z. Wahrscheinlichkeitstheorie und Verw. Gebiete 37 (1977), no. 3, 243-251.

18. Kiyosi Itô, Poisson point processes attached to Markov processes, Proceedings of the Sixth Berkeley Symposium on Mathematical Statistics and Probability (Univ. California, Berkeley, Calif., 1970/1971), Vol. III: Probability theory (Berkeley, Calif.), Univ. California Press, 1972, pp. 225-239.

19. Jörg-Uwe Löbus, A stationary Fleming-Viot type Brownian particle system, Mathematische Zeitschrift (2009), to appear.

20. Bernard Maisonneuve, Exit systems, Ann. Probability 3 (1975), no. 3, 399-411.

21. S. P. Meyn and R. L. Tweedie, Markov chains and stochastic stability, Communications and Control Engineering Series, Springer-Verlag London Ltd., London, 1993.

22. Thierry Meyre and Wendelin Werner, On the occupation times of cones by Brownian motion, Probab. Theory Related Fields 101 (1995), no. 3, 409-419.

23. Bernt Øksendal, Stochastic differential equations, sixth ed., Universitext, SpringerVerlag, Berlin, 2003, An introduction with applications.

Instytut Matematyki, Uniwersytet Mari SkŁodowskiej-Curie, 20-031 Lublin, Poland

Department of Mathematics, Box 354350, University of Washington, Seattle, WA 98195, USA 
Mathematics Institute, University of Warwick, Coventry CV4 7AL, United Kingdom

E-mail address: mariusz.bieniek@poczta.umcs.lublin.pl

E-mail address: burdzy@math. washington.edu

E-mail address: S.T.J.Finch@warwick.ac.uk 